\title{
CLIFFORD ALGEBRAS AS UNIFIED LANGUAGE FOR IMAGE PROCESSING AND PATTERN RECOGNITION
}

Valeriy Labunets

Urals State Tecnical University

Ekaterinburg, Russia

lab@rtf.ustu.ru

How wonderful are the things the Lord does! All who are delighted with them want to understand them.

—Good News Bible, Psalm 111:2

\begin{abstract}
The main goal of the paper is to show that commutative hypercomplex algebras and Clifford algebras can be used to solve problems of multicolor image processing and pattern recognition in a natural and effective manner.
\end{abstract}

Keywords: Clifford algebra, color images, hyperspectral images, invariants, multicolor images, pattern recognition.

\section{Introduction}

The concept of color and multispectral image recognition connects the topics we consider in this work. The term "multispectral (multicolor, multicomponent) image" is defined for an image with more than one component. An RGB image is an example of a color image featuring three separate image components $\mathrm{R}$ (red), G(green), and B(blue). We know that primates and animals with different evolutionary histories 
have color visual systems of different dimensionality. For example, the human brain uses three channel (RGB) images to recognize color (RGB)images. Primates have dichromacy and trichromacy visual systems, and they can use various $2 \mathrm{D}$ and $3 \mathrm{D}$ channels for the same purpose. Nonprimates have monochromacy and dichromacy visual systems. Images of such systems are real - or complex - valued functions. Reptiles have multichromacy visual systems. For example, the tortoise visual system has five types of color photoreceptors (R,G,B, DC,UV). Shrimps have the biggest known dimension of the visual system. They use ten spectral types of photoreceptors in their eyes to recognize fine spectral details. Our approach to multicolor image processing is to use so-called multiplet (multicolor or hypercomplex) numbers [1]-[7] to describe multicolor images and to operate directly on multi-channel images as on single-channel multiplet-valued images. In the classical approach every multicolor pixel (in particular, color pixel) is associated to a point of a $k \mathrm{D}$ multicolor vector space (to a point of a $3 \mathrm{D}$ RGB vector space for color images). In our approach, each image multicolor pixel is considered not as a $k \mathrm{D}$ vector, but as a multiplet (triplet) number, and multicolor (color) space is identified with the so-called multiplet (triplet) algebra. Note that both these assumptions (vector and hypercomplex natures of multicolor images) are only hypotheses. We have no biological evidence in the form of experiments that would verify that the brain actually uses any of the algebraic properties arising from structures of the vector spaces or the multiplet (triplet) algebra. It is our aim to show that the use of Clifford algebras fits more naturally to the tasks of multicolor image processing and recognition of multicolor patterns than does the use of color vector spaces. We give algebraic models of animals' visual systems using different hypercomplex and Clifford algebras. Our hypotheses are

1 Brains of primates operate with hypercomplex numbers during image processing and recognition.

2 Brains use different algebras on two levels (retina and Visual Cortex) for two general goals: image processing and pattern recognition, respectively. Multicolor images appear on the retina as functions with values in a multiplet $k \mathrm{D}$ algebra ( $k$-cycle algebra) where $k$ is the number of image spectral channels. But multicolor images in an animals' Visual Cortex (VC) are functions with values in a $2^{k}$-D Clifford algebra.

3 Visual systems of animals with different evolutionary histories use different hypercomplex algebras for color and multicolor image processing. 
One of the main and interesting problems of information science is clarification of how animals' eyes and brain recognize objects in the real world. Practice shows that they successfully cope with this problem and recognize objects at different locations, of different views and illumination, and with different degrees of blurring. But how is it done by the brain? How do we see? How do we recognize moving and changing objects of the surrounding world? A moving object is fixed in the retina as a sequence of different images. As in the famous aphorism of Heraclitus, who pointed out that one cannot step into the same river twice, we literally never see the same object twice. No individual image allows reaching a conclusion about the true shape of the object. This means that a set of sequential images appearing in the retina must contain a constant "something," thanks to which we see and recognize the object as a whole. This constant "something" is called invariant. For example, all letters ' $\mathrm{F}$ ' in Fig. 1 we interpret as the same for different geometric distortions. This fact means that all geometrically

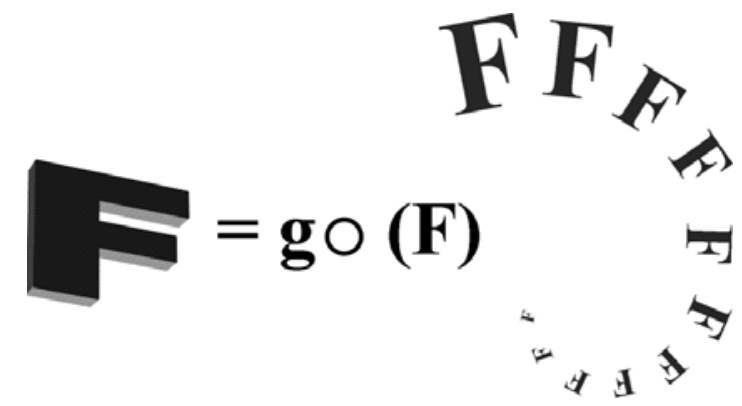

Figure 1. Geometrical distorted versa of letter ' $\mathrm{F}$ '

distorted letters ' $\mathrm{F}$ ' contain invariant features, which are not changed, when the shape of ' $F$ ' is changed. Our brain can extract these invariant features. In Fig. 2 we see hyperbolic (non-Euclidean) motions of grey-level mice and color fish. All transformed figures are interpreted as being the same. This fact means that all figures contain invariant features with respect to hyperbolic motions (and color transformations), and our brain can extract these invariant features from images, too. So, we see, we live in 3D Euclidean space but our brain can calculate invariants of images with respect to non-Euclidean transformations. In order for an artificial pattern recognition system to perform in the same way as any biological visual system, the recognition result should be invariant with respect to various transformation groups of the patterns such as translation, rotation, size variation, and change in illumination and 

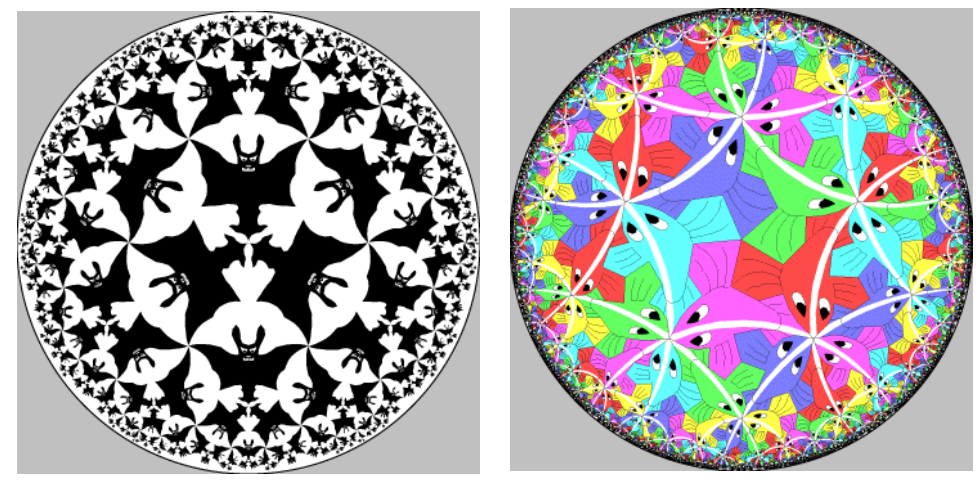

Figure 2. Non-Euclidean motions

color. The present work describes new methods of image recognition based on an algebraic-geometric theory of invariants. In this approach, each color or multicolor pixel is considered not as a $k \mathrm{D}$ vector, but as a $k \mathrm{D}$ hypercomplex number ( $k$ is the number of image spectral channels). Changes in the surrounding world which cause object shape and color transformations are treated not as matrix transforms, but as the action of some Clifford numbers in physical and perceptual spaces. We shall present some clues that Nature gives us about the role and importance of computing with hypercomplex numbers. We wish to review some of the reasons why such a state of affairs is necessary from a computational point of view. One can argue that Nature has also learned to utilize (through evolution) properties of hypercomplex numbers. Thus, the Visual Cortex might have the ability to operate as a Clifford algebra device. We don't agree with KRONECKER that "The Lord created the integers and the rest is the work of man." We think that the Lord knew Clifford algebras, and he was the first engineer who used these algebras to design animals' visual systems.

\section{Clifford algebras as models of physical spaces}

\subsection{Algebras of physical spaces}

We suppose that a brain calculates some hypercomplex-valued invariants of an image when recognizing it. Hypercomplex algebras generalize the algebras of complex numbers, quaternions and octonions. Of course, the algebraic nature of hypercomplex numbers must correspond to the spaces with respect to geometrically perceivable properties. For recognition of $2 \mathrm{D}, 3 \mathrm{D}$ and $n \mathrm{D}$ images we turn the spaces $\mathbf{R}^{2}, \mathbf{R}^{3}$ and $\mathbf{R}^{n}$ into 
corresponding algebras of hypercomplex numbers. Let "small" $n \mathrm{D}$ space $\mathbf{R}^{n}$ be spanned by the orthonormal basis of $n$ space hyperimaginary units $I_{i}, i=1,2, \ldots n$. We assume

$$
I_{i}^{2}=\left\{\begin{aligned}
+1 & \text { for } i=1,2, \ldots, p \\
-1 & \text { for } i=p+1, p+2, \ldots, p+q, \\
0 & \text { for } i=p+q+1, p+q+2, \ldots, p+q+r=n,
\end{aligned}\right.
$$

and $I_{i} I_{j}=-I_{j} I_{i}$. Now, we construct the "big" $2^{n} \mathrm{D}$ hypercomplex space $\mathbf{R}^{2^{n}}$. Let $\mathbf{b}=\left(b_{1}, b_{2}, \ldots, b_{n}\right) \in \mathbf{B}_{2}^{n}$ be an arbitrary n-bit vector, where $b_{i} \in \mathbf{B}_{2}=\{0,1\}$ and $\mathbf{B}_{2}^{n}$ is the $n \mathrm{D}$ Boolean algebra. Let us introduce $\mathbf{I}^{\mathbf{b}}:=I_{1}^{b_{1}} I_{2}^{b_{2}} \cdots I_{n}^{b_{n}}$. Then $2^{n}$ elements $\mathbf{I}^{\mathbf{b}}$ form a basis of $2^{n} \mathrm{D}$ space, i.e., for all $\mathcal{C} \in \mathbf{R}^{2^{n}}$ we have $\mathcal{C}:=\sum_{\mathbf{b} \in \mathbf{B}_{2}^{n}} c_{\mathbf{b}} \mathbf{I}_{\mathbf{b}}$. If $\mathcal{C}_{1}, \mathcal{C}_{2} \in \mathbf{R}^{2^{n}}$, then we can define their product $\mathcal{C}_{1} \mathcal{C}_{2}$. There are $3^{n}$ possibilities for $I_{i}^{2}=+1,0,-1$, $\forall i=1,2, \ldots, n$. Every possibility generates one algebra. Therefore, the space $\mathbf{R}^{2^{n}}$ with $3^{n}$ rules of the multiplication forms $3^{n}$ different $2^{n} \mathrm{D}$ algebras, which are called the space Clifford algebras [8]. We denote these algebras by $\mathcal{A}_{2^{n}}^{S p(p, q, r)}\left(\mathbf{R} \mid I_{1}, \ldots, I_{n}\right), \mathcal{A}_{2^{n}}^{S p(p, q, r)}$ or $\mathcal{A}_{2^{n}}^{S p}$, if $I_{1}, \ldots, I_{n}$, $p, q, r$ are fixed.

EXAMPLE 1 We start with the space $\mathbf{R}^{2}$ and provide it with the algebraic frame of algebras of generalized complex numbers: $\mathbf{R}^{2} \rightarrow \mathcal{A}_{2}(\mathbf{R}):=$ $\mathbf{R}+\mathbf{R} I=\left\{\mathbf{z}=x_{1}+I x_{2} \mid x_{1}, x_{2} \in \mathbf{R}\right\}$, where $I$ is a generalized imaginary unit.

- If $I^{2}=-1$, i.e., $I=i$, then $\mathcal{A}_{2}(\mathbf{R} \mid i)=\mathbf{C O M}=\left\{x_{1}+i x_{2} \mid\right.$ $\left.x_{1}, x_{2} \in \mathbf{R} ; i^{2}=-1\right\}$ is the field of complex numbers.

- If $I^{2}=+1$, i.e., $I=e$, then $\mathcal{A}_{2}(\mathbf{R} \mid e)=\mathbf{D O U}=\left\{x_{1}+e x_{2} \mid\right.$ $\left.x_{1}, x_{2} \in \mathbf{R} ; e^{2}=1\right\}$ is the ring of double numbers.

- If $I^{2}=0$, i.e., $I=\varepsilon$, then $\mathcal{A}_{2}(\mathbf{R} \mid \varepsilon)=\mathbf{D U A}=\left\{x_{1}+\varepsilon x_{2} \mid\right.$ $\left.x_{1}, x_{2} \in \mathbf{R} ; \varepsilon^{2}=0\right\}$ is the ring of dual numbers.

EXAMPle 2 Quaternions, as constructed by Hamilton, form the $4 \mathrm{D}$ algebra $\mathcal{A}_{4}=\mathcal{A}_{4}(\mathbf{R})=\mathcal{A}_{4}(\mathbf{R} \mid 1, i, j, k)=\mathbf{R}+\mathbf{R} i+\mathbf{R} j+\mathbf{R} k$ spanned by 4 hyperimaginary units $1, i, j, k$. The identities $i^{2}=j^{2}=k^{2}=-1$, $i j=-j i=k$ are valid for these units. $i^{2}=j^{2}=k^{2}=\delta \in\{-1,0,1\}$ can be set. Here, the two latter values (0 and 1) result in non-classical quaternions that were proposed by Clifford [8]. Introducing notations $I, J, K$ for new hyperimaginary units, we get nine spatial algebras of generalized quaternions, $\mathcal{A}_{4}(\mathbf{R} \mid 1, I, J, K):=\mathcal{A}_{4}=\mathbf{R}+\mathbf{R} I+\mathbf{R} J+\mathbf{R} K$ depending on which of nine possibilities resulting from $I^{2} \in\{1,0,-1\}$, $J^{2} \in\{1,0,-1\}$ is valid for the two independent hyperimaginary units. 
Every generalized quaternion $\mathbf{q}$ has the unique representation of the form $\mathbf{q}=q_{0}+q_{1} I+q_{2} J+q_{3} K=S c(\mathbf{q})+\operatorname{Vec}(\mathbf{q})$, where $q_{0}, q_{1}, q_{2}, q_{3}$ are real numbers and $S c(\mathbf{q}):=q_{0}, \operatorname{Vec}(\mathbf{q}):=q_{1} I+q_{2} J+q_{3} K$ are scalar and vector parts of the quaternion $\mathbf{q}$, respectively.

We can make $\mathcal{A}_{2^{n}}^{S p}$, be a ranked and $\mathbf{Z} / 2 \mathbf{Z}$-graded algebra. Let $r(\mathbf{b})$ be the Hamming weight $\left(=\right.$ rank) of $\mathbf{b}$, i.e., a functional $r: \mathbf{B}_{2}^{n} \longrightarrow[0, n-1]$ defined by $r(\mathbf{b}):=\sum_{i=1}^{n} b_{i}$, and let $\partial(\mathbf{b})=r(\mathbf{b})(\bmod 2)$ be the $\operatorname{grad}$ of b. Then $\mathcal{A}_{2^{n}}^{S p}$ can be represented as the ranked and $\mathbf{Z} / 2 \mathbf{Z}$-graded sums $\mathcal{A}_{2^{n}}^{S p}=\bigoplus_{r=0}^{n} \mathcal{A}_{2^{n}}^{[r]}$ and $\mathbf{R}^{2^{n}}=\bigoplus_{\partial=0}^{1} \mathcal{A}_{2^{n}}^{\{\partial\}}$, where the dimension of the vector space $\mathcal{A}_{2^{n}}^{[k]}$ equals the binomial coefficient $C_{n}^{k}$ and $\sum_{k=0}^{n} C_{n}^{k}=2^{n}$. The dimensions of $\mathcal{A}_{2^{n}}^{\{0\}}$ and $\mathcal{A}_{2^{n}}^{\{1\}}$ are equal to $2^{n-1}$. The subspaces $\mathcal{A}_{2^{n}}^{[k]}$ are spanned by the $k$-products of units $I_{i_{1}} I_{i_{2}} \ldots I_{i_{k}}\left(i_{1}<i_{2}<\ldots<\right.$ $\left.i_{k}\right)$, i.e., by all basis vectors $\mathbf{I}_{\mathbf{b}}$ with $r(\mathbf{b})=k$. Every element $\mathcal{C}:=$ $\sum_{\mathbf{b} \in \mathbf{B}_{2}^{n}} c_{\mathbf{b}} \mathbf{I}_{\mathbf{b}}$ of $\mathcal{A}_{2^{n}}^{S p}$ has the representations: $\mathcal{C}=\mathfrak{C}^{[0]}+\mathfrak{C}^{[1]}+\ldots+\mathfrak{C}^{[n]}$ and $\mathcal{C}=\mathcal{C}^{\{0\}}+\mathcal{C}^{\{1\}}$, where $\mathcal{C}^{[0]} \in \mathcal{A}_{2^{n}}^{[0]}$ is the scalar part of the Clifford numbers, $\mathrm{C}^{[1]} \in \mathcal{A}_{2^{n}}^{[1]}$ is its vector part, $\mathrm{C}^{[2]} \in \mathcal{A}_{2^{n}}^{[2]}$ is its bivector part, ..., $\mathcal{C}^{[n]} \in \mathcal{A}_{2^{n}}^{[n]}$ is its $n$-vector part, and, finally, $\mathcal{C}^{\{0\}}$ and $\mathcal{C}^{\{1\}}$ are even and odd parts of the Clifford number $\mathcal{C}$. If $\mathcal{C} \in \mathcal{A}_{2^{n}}^{\{l\}}$, we put $\partial(\mathcal{C})=l$ and say that $l$ is the degree of $\mathcal{C}$. Multiplication of two Clifford numbers of ranks $k$ and $s$ gives the sum of Clifford numbers from $|k-s|$ to $p=\min (k+s, 2 n-k-s)$ with increment 2, i.e., $\mathcal{A}^{[k]} \mathcal{B}^{[s]}=\mathrm{C}^{[|k-s|]}+$ $\mathcal{C}^{[|k-s|+2]}+\ldots+\mathcal{C}^{[p]}$.

\subsection{Geometries of physical spaces}

The conjugation operation in $\mathcal{A}_{2^{n}}^{S p(p, q, r)}$ maps every Clifford number $\mathcal{C}:=c_{0} I_{0}+\sum_{\mathbf{b} \neq 0} c_{\mathbf{b}} \mathbf{I}_{\mathbf{b}}$ to the number $\overline{\mathcal{C}}=c_{0} I_{0}-\sum_{\mathbf{b} \neq 0} c_{\mathbf{b}} \mathbf{I}_{\mathbf{b}}$. The algebras $\mathcal{A}_{2^{n}}^{S p(p, q, r)}$ are transformed into $2^{n} \mathrm{D}$ pseudometric spaces designated by $\mathcal{C} \mathcal{L}_{2^{n}}^{S p(p, q, r)}$ or $\mathcal{C} \mathcal{L}_{2^{n}}^{p, q, r}$, if the pseudodistance between two Clifford numbers $\mathcal{A}$ and $\mathcal{B}$ is defined by $\rho(\mathcal{A}, \mathcal{B})=\sqrt{(\mathcal{A}-\mathcal{B}) \overline{(\mathcal{A}-\mathcal{B})}}$. Subspaces of pure vector Clifford numbers $x_{1} I_{1}+\ldots+x_{n} I_{n} \in \operatorname{Vec}^{1}\left(\mathcal{A}_{2^{n}}^{S p}\right)$ are $n \mathrm{D}$ spaces $\mathbf{R}^{n}:=\mathcal{G} \mathcal{R}_{n}^{S p(p, q, r)}$. The pseudometrics constructed in $\mathcal{C} \mathcal{L}_{2^{n}}^{S p(p, q, r)}$ induce corresponding pseudometrics in $\mathcal{G R}_{n}^{S p(p, q, r)}$.

EXAMPLE 3 In $\mathcal{A}_{2}(\mathbf{R})$ we introduce a conjugation operation which maps every element $\mathbf{z}=x_{1}+I x_{2}$ to the element $\overline{\mathbf{z}}=x_{1}-I x_{2}$. Now, the generalized complex plane is turned into the pseudometric space $\mathcal{A}_{2}(\mathbf{R}) \longrightarrow$ 
$\mathcal{G C}_{2}^{S p(p, q, r)}$ if one defines the pseudodistance as:

$$
\rho\left(\mathbf{z}_{1}, \mathbf{z}_{2}\right)= \begin{cases}\sqrt{\left(x_{2}-x_{1}\right)^{2}+\left(y_{2}-y_{1}\right)^{2}}, & \text { if } \mathbf{z} \in \mathcal{A}_{2}(\mathbf{R} \mid i), \\ \sqrt{\left(x_{2}-x_{1}\right)^{2}-\left(y_{2}-y_{1}\right)^{2}}, & \text { if } \mathbf{z} \in \mathcal{A}_{2}(\mathbf{R} \mid e), \\ \left|x_{2}-x_{1}\right|, & \text { if } \mathbf{z} \in \mathcal{A}_{2}(\mathbf{R} \mid \varepsilon),\end{cases}
$$

where $\mathbf{z}_{1}:=\left(x_{1}, x_{2}\right)=x_{1}+I x_{2}, \mathbf{z}_{2}:=\left(y_{1}, y_{2}\right)=y_{1}+I y_{2}$. So, the plane of the classical complex numbers is the $2 D$ Euclidean space $\mathcal{G e}_{2}^{S p(2,0,0)}$, the double numbers plane is the $2 D$ Minkowskian space $\mathcal{G C}_{2}^{S p(1,1,0)}$, and the dual numbers plane is the $2 D$ Galilean space $\mathcal{G C}_{2}^{S p(1,0,1)}$. When one speaks about all three algebras (or geometries) simultaneously, then the corresponding algebra (or geometry) is that of generalized complex numbers, denoted by $\mathcal{A}_{2}^{S p(p, q, r)}$ (or $\left.\mathcal{G C}_{2}^{S p(p, q, r)}\right)$.

EXAMPLE 4 In $\mathcal{A}_{4}(\mathbf{R})$ we introduce a conjugation operation which maps every quaternion $\mathbf{q}=q_{0}+I q_{1}+J q_{2}+K q_{3}$ to the element $\bar{q}=q_{0}-$ $I q_{1}-J q_{2}-K q_{3}$. If the pseudodistance $\rho(\mathbf{p}, \mathbf{q})$ between two generalized quaternions $\mathbf{p}$ and $\mathbf{q}$ is defined as the modulus of their difference $\mathbf{u}=\mathbf{p}-\mathbf{q}=t+x I+y J+z K: \rho(\mathbf{p}, \mathbf{q})=\sqrt{(\mathbf{p}-\mathbf{q}) \overline{(\mathbf{p}-\mathbf{q})}}=$ $\sqrt{\mathbf{u} \overline{\mathbf{u}}}$, then nine spatial algebras $\mathcal{A}_{4}(\mathbf{R})$ are transformed into nine $4 D$ pseudometric spaces designed as $\mathcal{G H}_{4}^{S p(p, q, r)}$, where $p, q$ and $r$ stand for the number of basis vectors with squares $1,-1$ and 0 , respectively, and $p+q+r=n$. Thus, the pseudodistance can take positive, negative and pure imaginary values. There are only 5 different geometries $\mathcal{G H}_{4}^{S p(p, q, r)}$ : $\mathcal{G H}_{4}^{S p(4,0,0)}, \mathcal{G H}_{4}^{S p(2,2,0)}, \mathcal{G H}_{4}^{S p(2,0,2)}, \mathcal{G H}_{4}^{S p(1,3,0)}, \mathcal{G H}_{4}^{S p(1,2,1)}$. The subspaces of pure vector-valued generalized quaternions $x I+y J+z K$ are $3 D$ spaces $\mathcal{G R}_{3}^{S(p, q, r)}:=\operatorname{Vec}\left\{\mathcal{G H}_{4}^{S p(p, q, r)}\right\}$. The pseudometrics introduced in $\mathcal{G H}_{4}^{S p(p, q, r)}$ induce only three different pseudometrics in $\mathcal{G R}_{3}^{S p(p, q, r)}$ :

$\rho(\operatorname{Vec}\{\mathbf{p}\}, \operatorname{Vec}\{\mathbf{q}\})=|\operatorname{Vec}\{\mathbf{p}-\mathbf{q}\}|=|\operatorname{Vec}\{\mathbf{u}\}|=\left\{\begin{array}{l}\sqrt{x^{2}+y^{2}+z^{2}}, \\ \sqrt{x^{2}-y^{2}-z^{2}}, \\ \sqrt{x^{2}}=|x| .\end{array}\right.$

They will be denoted by $\mathcal{G R}_{3}^{S p(3,0,0)} \mathcal{G R}_{3}^{S p(1,0,2)}, \mathcal{G R}_{3}^{S p(1,2,0)}$. They form Euclidean, Minkowskian, and Galilean 3D pseudometric spaces, respectively.

All even Clifford numbers $\mathcal{E}_{0} \in \mathcal{A}_{2^{n}}^{\{0\}}$ of unit modulus represent the rotation group of the corresponding space $\mathcal{G R}_{n}^{S p(p, q, r)}$, which is called the spinor group and is denoted by $\operatorname{Spin}\left(\mathcal{A}_{2^{n}}^{S p(p, q, r)}\right)$. Generalized complex 
numbers and quaternions of unit modulus have the forms: $\mathbf{e}_{0}=e^{I \varphi}=$ $\cos \varphi+I \sin \varphi, Q_{0}=e^{\mathbf{u}_{0} \varphi}=\cos \varphi+\mathbf{u}_{0} \sin \varphi$, where $\cos \varphi$ and $\sin \varphi$ are trigonometric functions in the corresponding $n \mathrm{D} \mathcal{G C}_{n}^{S p(p, q, r)}$-geometries, $\varphi$ is a rotation angle around the vector-valued quaternion $\mathbf{u}_{0}$ of unit modulus $\left(\left|\mathbf{u}_{0}\right|=1, \mathbf{u}_{0}=-\overline{\mathbf{u}_{0}}\right)$. Clifford numbers $\mathcal{E}_{0} \in \operatorname{Spin}\left(\mathcal{A}_{2^{n}}^{S p(p, q, r)}\right)$ with unit modulus have the analogous form $\mathcal{E}_{0}=e^{\mathbf{u}_{0} \varphi}=\cos \varphi+\mathbf{u}_{0} \sin \varphi \in$ $\operatorname{Spin}\left(\mathcal{A}_{2^{n}}^{S p(p, q, r)}\right)$ for the appropriate bivector $\mathbf{u}_{0}$.

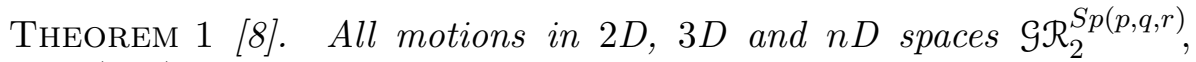
$\mathcal{G R}_{3}^{S p(p, q, r)}, \mathcal{G R}_{n}^{S p(p, q, r)}$ are represented in the forms:

$$
\mathbf{z}^{\prime}=\mathbf{e}_{0} \mathbf{z} \mathbf{e}_{0}+\mathbf{w}, \quad \mathbf{x}^{\prime}=\mathcal{Q}_{0} \mathbf{x} \mathcal{Q}_{0}^{-1}+\mathbf{w}, \quad \mathbf{x}^{\prime}=\mathcal{E}_{0} \mathbf{x} \mathcal{E}_{0}^{-1}+\mathbf{w},
$$

where $\mathbf{e}_{0}=e^{I \varphi / 2}, Q_{0}=e^{\mathbf{u}_{0} \varphi / 2}, \mathcal{E}_{0}=e^{\mathbf{u}_{0} \varphi / 2}$, and $\left|\mathbf{e}_{0}\right|=\left|Q_{0}\right|=\left|\mathcal{E}_{0}\right|=1$. If $\left|\mathbf{e}_{0}\right|,\left|Q_{0}\right|,\left|\mathcal{E}_{0}\right| \neq 1$, then the latter transformations form the "small" affine groups $\operatorname{Aff}\left(\mathcal{G R}_{2}^{S p(p, q, r)}\right), \operatorname{Aff}\left(\mathcal{G R}_{3}^{S p(p, q, r)}\right), \operatorname{Aff}\left(\mathcal{G R}_{n}^{S p(p, q, r)}\right)$, respectively.

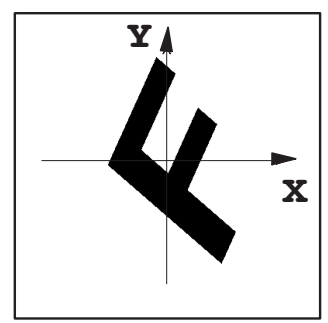

a)

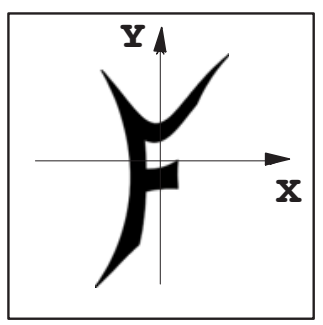

b)

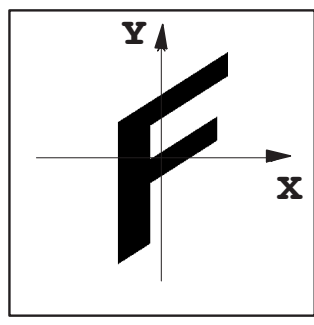

C)

Figure 3. Rotations in a) $2 \mathrm{D}$ Euclidean space $\mathcal{G C}_{2}^{S p(2,0,0)}$, b) $2 \mathrm{D}$ Minkowskian space $\mathcal{G C}_{2}^{S p(1,1,0)}$ and c) $2 \mathrm{D}$ Galilean space $\mathcal{G C}_{2}^{S p(1,0,1)}$.

Using this theorem, we can describe geometric distortions of images in the language of Clifford algebras. These distortions will be caused by: 1) $n \mathrm{D}$ translations $\mathbf{x} \longrightarrow \mathbf{x}+\mathbf{w}$; 2) $n \mathrm{D}$ rotations $\mathbf{x} \longrightarrow \mathcal{E}_{0}(\mathbf{x}+\mathbf{w}) \mathcal{E}_{0}^{-1}$; 3) dilatation: $\mathbf{x} \longrightarrow \lambda \mathbf{x}$, where $\lambda \in \mathbf{R}^{+}$. If $f(\mathbf{x})$ is an initial image and $\lambda \mathcal{E}_{0} \mathbf{w} \mathbf{f}(\mathbf{q})$ is its distorted version, then ${ }_{\lambda \mathcal{E}_{0} \mathbf{w}} \mathbf{f}(\mathbf{x}):=\mathbf{f}\left(\lambda \mathcal{E}_{0}(\mathbf{x}+\mathbf{w}) \mathcal{E}_{0}^{-1}\right)$, where $\lambda$ is a scale factor, $\mathbf{x}, \mathbf{w} \in \mathcal{G R}_{n}^{S p(p, q, r)}$. We suppose that the human brain can use the spinor and "small" affine groups for mental rotations (see Fig. 3) and motions of images (for example, in a dream), which are contained in the brain memory on the so-called "screen of mind." 


\section{Clifford Algebras as Models of Perceptual Multicolor Spaces}

Early in the 19th century Young (1802) proposed that the human visual system contains three color mechanisms. This theory was later supported by Helmholtz (1852) and became known as the YoungHelmholtz theory of color vision. Later, HERING (1878) proposed that color vision is mediated by red-green and blue-yellow opponent mechanisms. Thus, for a time it appeared there were two conflicting theories of color vision. As a result of experimental work, it has since become recognized that the two theories describe different levels in the visual system. In agreement with the Young-Helmholtz theory, there are three photoreceptor mechanisms, i.e., cone types, in the human retina, and, in accordance with Hering's theory, there are color-opponent neurons at higher levels in the VC. The multicomponent color image appears on the retina as the $k \mathrm{D}$ vector

$$
\mathbf{f}_{M c o l}^{\text {Ret }}(\mathbf{x})=\left[\begin{array}{c}
f_{1}(\mathbf{x}) \\
f_{2}(\mathbf{x}) \\
\ldots \\
f_{k}(\mathbf{x})
\end{array}\right]=\left[\begin{array}{c}
\int_{\lambda} S^{o b j}(\lambda, \mathbf{x}) H_{1}(\lambda) d \lambda \\
\int_{\lambda} S^{o b j}(\lambda, \mathbf{x}) H_{2}(\lambda) d \lambda \\
\cdots \\
\int_{\lambda} S^{o b j}(\lambda, \mathbf{x}) H_{k}(\lambda) d \lambda
\end{array}\right],
$$

where $S^{o b j}(\lambda, \mathbf{x})$ is the color spectrum received from the point $\mathbf{x}$ of an object and $H_{1}(\lambda), H_{2}(\lambda), \ldots, H_{k}(\lambda)$ are sensor sensitivity functions. We give algebraic models for two levels (retina and VC) of visual systems using different hypercomplex $\mathbf{Z} / k \mathbf{Z}$-graded Clifford algebras.

\subsection{Algebraization of the Young-Helmholtz model}

3.1.1 The Young-Helmholtz model of color images. We shall represent RGB-color images that appear on the human retina as triplet-valued functions: $\mathbf{f}_{c o l}^{R e t}(\mathbf{x})=f_{R}(\mathbf{x}) 1_{c o l}+f_{G}(\mathbf{x}) \varepsilon_{c o l}+f_{B}(\mathbf{x}) \varepsilon_{c o l}^{2}$, where $1_{c o l}, \varepsilon_{c o l}^{1}, \varepsilon_{c o l}^{2}$ are hyperimaginary units, $\varepsilon_{c o l}^{3}=1$. Numbers of the form $\mathcal{C}=x 1+y \varepsilon_{c o l}+z \varepsilon_{c o l}^{2}\left(\varepsilon_{c o l}^{3}=1\right)$ were considered by GREAVES [10]. According to Greaves, these numbers are called the triplet or 3-cycle numbers. We shall call them the color numbers. The product of two color numbers $\mathcal{C}_{1}=a_{0}+a_{1} \varepsilon_{c o l}+a_{2} \varepsilon_{c o l}^{2}$ and $\mathcal{C}_{2}=b_{0}+b_{1} \varepsilon_{c o l}+b_{2} \varepsilon_{c o l}^{2}$ is given by

$$
\begin{gathered}
\mathcal{C}_{1} \mathcal{C}_{2}=\left(a_{0}+a_{1} \varepsilon_{c o l}+a_{2} \varepsilon_{c o l}^{2}\right)\left(b_{0}+b_{1} \varepsilon_{c o l}+b_{2} \varepsilon_{c o l}^{2}\right)= \\
\left(a_{0} b_{0}+a_{1} b_{2}+a_{2} b_{1}\right)+\left(a_{1} b_{0}+a_{2} b_{2}+a_{0} b_{1}\right) \varepsilon_{c o l}+\left(a_{2} b_{0}+a_{1} b_{1}+a_{2} b_{0}\right) \varepsilon_{c o l}^{2} .
\end{gathered}
$$

Thus, the color product is isomorphic to the 3-point cyclic convolution. The color conjugate $\overline{\mathcal{C}}$ of a color number $\mathcal{C}=x+y \varepsilon_{c o l}+z \varepsilon_{c o l}^{2}$ is defined 
by $\overline{\mathcal{C}}=\overline{x+y \varepsilon_{c o l}+z \varepsilon_{c o l}^{2}}=x+y \bar{\varepsilon}_{c o l}+z \bar{\varepsilon}_{c o l}^{2}=x+z \varepsilon_{c o l}+y \varepsilon_{c o l}^{2}$. The norm $\|\mathrm{C}\|=\mathfrak{e} \overline{\mathcal{C}}$ is given by $\|\mathrm{C}\|^{2}=\mathfrak{e} \overline{\mathcal{C}}=\left(x^{2}+y^{2}+z^{2}\right)-(x y+x z+y z)$. Each color number has three modules

$$
\begin{gathered}
\|\mathcal{C}\|_{1}=|x+y+z|, \quad\|\mathcal{C}\|_{2}=\sqrt{x^{2}+y^{2}+z^{2}-x y-x z-y z} \\
\|\mathcal{C}\|_{3}=\sqrt[3]{x^{3}+y^{3}+z^{3}-3 x y z}
\end{gathered}
$$

possessing the properties: $\left\|\mathcal{C}_{1} \mathfrak{C}_{2}\right\|_{i}=\left\|\mathcal{C}_{1}\right\|{ }_{i}\left\|\mathcal{C}_{2}\right\|_{i}, i=1,2,3$ and $\|\mathcal{C}\|_{3}^{3}=$ $\|\mathcal{C}\|_{2}^{2}\|\mathcal{C}\|_{1}$. Triplets, strictly speaking, do not form a 3D field, but form an associative so-called triplet (color) algebra

$$
\mathcal{A}_{3}^{c o l}=\mathcal{A}_{3}^{c o l}(\mathbf{R})=\mathcal{A}_{3}\left(\mathbf{R} \mid 1, \varepsilon_{c o l}, \varepsilon_{c o l}^{2}\right):=\mathbf{R} 1+\mathbf{R} \varepsilon_{c o l}+\mathbf{R} \varepsilon_{c o l}^{2} .
$$

Greaves [10] considered a color number $x+y \varepsilon+z \varepsilon^{2}$ (here, $\varepsilon \equiv \varepsilon_{c o l}$ )

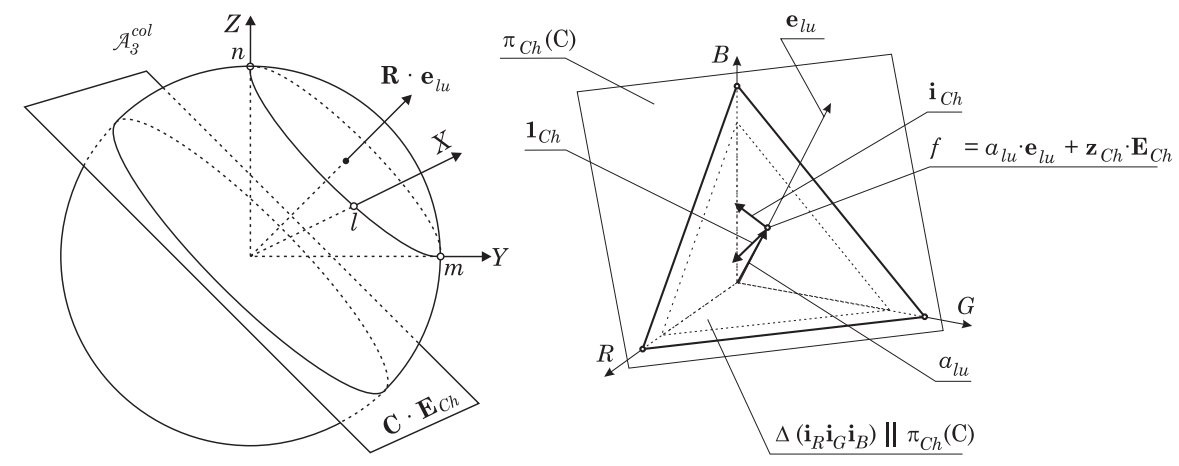

Figure 4. Geometric interpretation of triplet algebra

as a point of $3 \mathrm{D}$ space and gave the following geometric interpretation of triplet operations. He constructed a sphere (see Fig. 4) with the center at the origin and denoted intersection points of the sphere with the coordinate axes $X, Y, Z$ in the positive direction by $l, m, n$, respectively. He drew a circle via points $l, m, n$; the "symmetrical axis" $\mathbf{e}_{l u}$ was drawn via the center of this circle and the origin; the "symmetrical plane" $\mathbf{E}_{c h}$ is perpendicular to the axis $\mathbf{e}_{l u}$. Then Greaves considered rectangular projections of color numbers $\mathcal{C}$ on the axis $\mathbf{e}_{l u}$ and plane $\mathbf{E}_{c h}$. Furthermore, he proved that every triplet $\mathcal{C}$ can be represented as a sum of a real number being depicted by a rectangular projection of the triplet on the axis $\mathbf{e}_{l u}$ and a complex number being depicted by a rectangular projection of the triplet on the plane $\mathbf{E}_{c h}$. Therefore, the color algebra is the direct sum of the real $\mathbf{R}$ and complex $\mathbf{C}$ fields: $\mathcal{A}_{3}(\mathbf{R}, \mathbf{C})=\mathbf{R} \cdot \mathbf{e}_{l u}+\mathbf{C} \cdot \mathbf{E}_{c h}=\mathbf{R} \oplus \mathbf{C}$, where $\mathbf{e}_{l u}:=\left(1+\varepsilon+\varepsilon^{2}\right) / 3$, 
$\mathbf{E}_{c h}:=\left(1+\omega_{3} \varepsilon^{2}+\omega_{3}^{2} \varepsilon\right) / 3$ are orthogonal "real" and "complex" idempotents $\left(\mathbf{e}_{l u}^{2}=\mathbf{e}_{l u}, \mathbf{E}_{c h}^{2}=\mathbf{E}_{c h}, \mathbf{e}_{l u} \mathbf{E}_{c h}=\mathbf{E}_{c h} \mathbf{e}_{l u}=0\right)$, respectively, and $\omega_{3}:=e^{\frac{2 \pi i}{3}}$. Therefore, every color number $\mathcal{C}=x+y \varepsilon^{1}+z \varepsilon^{2}$ is a linear combination $\mathcal{C}=x+y \varepsilon^{1}+z \varepsilon^{2}=a_{l u} \cdot \mathbf{e}_{l u}+\mathbf{z}_{c h} \cdot \mathbf{E}_{c h}=\left(a_{l u}, \mathbf{z}_{c h}\right)$ of the "scalar" $a_{l u} \mathbf{e}_{l u}$ and "complex" parts $\mathbf{z}_{c h} \mathbf{E}_{c h}$ in the idempotent basis $\left\{\mathbf{e}_{l u}, \mathbf{E}_{c h}\right\}$. We will call the real numbers $a_{l u} \in \mathbf{R}$ the luminance (intensity) numbers, and the complex numbers $\mathbf{z}_{c h}=b+j c \in \mathbf{C}$ the chromaticity numbers. Obviously, $a_{l u} \mathbf{e}_{l u}:=\mathrm{Ce}_{l u}=\left(x+y \varepsilon^{1}+z \varepsilon^{2}\right) \mathbf{e}_{l u}=$ $(x+y+z) \mathbf{e}_{l u}, \mathbf{z}_{c h} \mathbf{E}_{c h}:=\mathcal{e} \mathbf{E}_{c h}=\left(x+y \varepsilon^{1}+z \varepsilon^{2}\right) \mathbf{E}_{c h}=\left(x+\omega_{3}^{1} y+z \omega_{3}^{2}\right) \mathbf{E}_{c h}$, where $a_{l u}=x+y+z, \quad \mathbf{z}_{c h}=x+\omega_{3}^{2} y+z \omega_{3}^{1}$. In the new representation two main arithmetic operations have very simple forms:

$$
\begin{gathered}
\mathcal{C}_{1} \pm \mathcal{C}_{2}=\left(a_{1} \mathbf{e}_{l u}+\mathbf{z}_{1} \mathbf{E}_{c h}\right) \pm\left(a_{2} \mathbf{e}_{l u}+\mathbf{z}_{2} \mathbf{E}_{c h}\right)=\left(a_{1} \pm a_{2}\right) \mathbf{e}_{l u}+\left(\mathbf{z}_{1} \pm \mathbf{z}_{2}\right) \mathbf{E}_{c h} \\
\mathcal{C}_{1} \mathcal{C}_{2}=\left(a_{1} \mathbf{e}_{l u}+\mathbf{z}_{1} \mathbf{E}_{c h}\right)\left(a_{2} \mathbf{e}_{l u}+\mathbf{z}_{2} \mathbf{E}_{c h}\right)=\left(a_{1} a_{2}\right) \mathbf{e}_{l u}+\left(\mathbf{z}_{1} \mathbf{z}_{2}\right) \mathbf{E}_{c h}
\end{gathered}
$$

or, briefly, $\mathcal{C}_{1} \pm \mathcal{C}_{2}=\left(a_{1}, \mathbf{z}_{1}\right) \pm\left(a_{2}, \mathbf{z}_{2}\right)=\left(a_{1} \pm a_{2}, \mathbf{z}_{1} \pm \mathbf{z}_{2}\right), \mathcal{C}_{1} \mathfrak{C}_{2}=$ $\left(a_{1}, \mathbf{z}_{1}\right) \cdot\left(a_{2}, \mathbf{z}_{2}\right)=\left(a_{1} a_{2}, \mathbf{z}_{1} \mathbf{z}_{2}\right)$. For $\overline{\mathcal{C}}$ we have $\overline{\mathcal{C}}=\overline{x+y \varepsilon^{1}+z \varepsilon^{2}}=$ $a_{l u} \cdot \mathbf{e}_{l u}+\overline{\mathbf{z}}_{c h} \cdot \mathbf{E}_{c h}=\left(a_{l u}, \overline{\mathbf{z}}_{c h}\right)$. Therefore,

$$
\begin{gathered}
\|\mathcal{C}\|_{1}=|x+y+z|=\left|a_{l u}\right|, \quad\|\mathcal{C}\|_{2}^{2}=\left|x^{2}+y^{2}+z^{2}-x y-x z-y z\right|=\left\|\mathbf{z}_{c h}\right\|^{2}, \\
\|\mathcal{C}\|_{3}^{3}=\left|x^{3}+y^{3}+z^{3}-3 x y z\right|=\left|a_{l u}\right| \mid \mathbf{z}_{c h} \|^{2} .
\end{gathered}
$$

The norms $\|.\|_{1},\|.\|_{2}$ are called the luminance and chromaticity norms, respectively. We can consider a color image in the two forms:

$$
\begin{gathered}
\mathbf{f}_{c o l}^{R e t}(x, y)=f_{R}(x, y) 1_{c o l}+f_{G}(x, y) \varepsilon_{c o l}+f_{B}(x, y) \varepsilon_{c o l}^{2}= \\
f_{l u}(x, y) \mathbf{e}_{l u}+\mathbf{f}_{c h}(x, y) \mathbf{E}_{c h} .
\end{gathered}
$$

In the second form we have separated the color image into two terms: the luminance (intensity) term $f_{l u}(x, y)$ and the chromacity term $\mathbf{f}_{c h}(x, y)$ (color information). This color transformation is a linear projection (see Fig. 4) of the color vector-valued pixel in the color space on the diagonal vector $\mathbf{e}_{l u}:=(1,1,1)=\mathbf{i}_{R}+\mathbf{i}_{G}+\mathbf{i}_{B}$ and on the 2 D plane $\pi_{c h}(\mathbf{C})$ which is orthogonal to the diagonal vector $\mathbf{e}_{l u}: \pi_{c h}(\mathbf{C}) \perp \mathbf{e}_{l u}$. The vector $\mathbf{e}_{l u}$ is called the luminance (white) vector and the $2 \mathrm{D}$ plane $\pi_{c h}(\mathbf{C})$ is called the chromacy plane of RGB-space. The triangle $\triangle\left(\mathbf{i}_{R} \mathbf{i}_{G} \mathbf{i}_{B}\right) \| \pi_{c h}(\mathbf{C})$ drawn between three primaries $\mathbf{i}_{R} \mathbf{i}_{G} \mathbf{i}_{B}$ is called the Maxwell triangle. The plain $\pi_{c h}(\mathbf{C})$ is equipped with the structure of the complex field $\mathbf{C}$. Therefore, we can consider an RGB image as a pair of images $\mathbf{f}_{c o l}^{R e t}(\mathbf{x})=$ $\left(f_{l u}(\mathbf{x}), \mathbf{f}_{c h}(\mathbf{x})\right)$, where $f_{l u}(\mathbf{x}):=\left\langle\mathbf{e}_{l u} \mid \mathbf{f}_{c o l}^{R e t}(\mathbf{x})\right\rangle=f_{R}(\mathbf{x})+f_{G}(\mathbf{x})+f_{B}(\mathbf{x})$, $\mathbf{f}_{c h}(\mathbf{x}):=\mathbf{f}_{c o l}(\mathbf{x})-f_{l u}(\mathbf{x}) \mathbf{e}_{l u}$, and $\left\langle f_{l u}(\mathbf{x}) \mid \mathbf{f}_{c h}(\mathbf{x})\right\rangle=0$. Here, $f_{l u}(\mathbf{x}):$ $\mathbf{R}^{2} \longrightarrow \mathbf{R}^{+}$is a real-valued image (grey-level image) and $\mathbf{f}_{c h}(\mathbf{x}): \mathbf{R}^{2} \longrightarrow$ 
$\pi_{c h}(\mathbf{C})$ is a complex-valued image. Hence, each pixel is represented both by a real number $f_{l u}(\mathbf{x})$ for the luminance and a complex number $\mathbf{f}_{c h}(\mathbf{x})$ for the chromatic information. The argument of the complex number $\mathbf{f}_{c h}(\mathbf{x})$ is directly equivalent to the traditional definition of hue, and the modulus of the complex number is similar to saturation. Changes in both the surrounding world and mental space of reality such as intensity, color or illumination can be treated in the triplet algebra language as the action of some transformation groups in the perceptual color space $\mathcal{A}_{3}^{\text {col }}$. Let us consider some of them.

1 Let $\mathcal{A}=\left(1, e^{i \varphi}\right)$. Then transformation of color images $\mathbf{f}_{\text {col }}^{\text {Ret }}(\mathbf{x}) \longrightarrow$ $\mathcal{A} \mathbf{f}_{c o l}(\mathbf{x})=\left(1, e^{i \varphi}\right) \cdot\left(f_{l u}(\mathbf{x}), \mathbf{f}_{c h}(\mathbf{x})\right)=\left(f_{l u}(\mathbf{x}), e^{i \varphi} \mathbf{f}_{c h}(\mathbf{x})\right)$ changes only the hue of the initial image. The set of all such transformations forms the hue orthogonal group $\mathbb{H} \mathbb{O G}(2):=\left\{\left(1, e^{i \varphi}\right) \mid e^{i \varphi} \in\right.$ C\}.

2 Now let $\mathcal{A}=(1, \lambda), \lambda>0$. Then transformation of color images $\mathbf{f}_{c o l}^{R e t}(\mathbf{x}) \longrightarrow \mathcal{A} \mathbf{f}_{c o l}(\mathbf{x})=(1, \lambda) \cdot\left(f_{l u}(\mathbf{x}), \mathbf{f}_{c h}(\mathbf{x})\right)=\left(f_{l u}(\mathbf{x}), \lambda \mathbf{f}_{c h}(\mathbf{x})\right)$ changes only the saturation of the initial image. The set of all such transformations forms the saturation dilation group $\mathbb{S D G}(2):=$ $\left\{(1, a) \mid a \in \mathbf{R}^{+}\right\}$.

3 If $\mathcal{A}=\left(1, \mathbf{z}_{c h}\right)=\left(1,\left|\mathbf{z}_{c h}\right| e^{i \varphi}\right)$, then transformations $\mathbf{f}_{\text {col }}^{\text {Ret }}(\mathbf{x}) \longrightarrow$ $\mathcal{A} \mathbf{f}_{c o l}(\mathbf{x})=\left(1,\left|\mathbf{z}_{c h}\right| e^{i \varphi}\right) \cdot\left(f_{l u}(\mathbf{x}), \mathbf{f}_{c h}(\mathbf{x})\right)=\left(f_{l u}(\mathbf{x}),\left|\mathbf{z}_{c h}\right| e^{i \varphi} \mathbf{f}_{c h}(\mathbf{x})\right)$ change both hue and saturation of the initial image. The set of all such transformations forms the chromatic group $\mathbb{C} \mathbb{G}(2):=$ $\left\{\left(1, \mathbf{z}_{c h}\right)=\left(1,|\mathbf{z}| e^{i \varphi}\right)|| \mathbf{z}_{c h} \mid e^{i \varphi} \in \mathbf{C}\right\}$.

4 If $\mathcal{A}=\left(a_{l u}, \mathbf{z}_{c h}\right)=\left(a_{l u},\left|\mathbf{z}_{c h}\right| e^{i \varphi}\right)$, where $a>0$, then transformations $\mathbf{f}_{c o l}^{\text {Ret }}(\mathbf{x}) \longrightarrow \mathcal{A} \mathbf{f}_{c o l}(\mathbf{x})=\left(a_{l u},\left|\mathbf{z}_{c h}\right| e^{i \varphi}\right) \cdot\left(f_{l u}(\mathbf{x}), \mathbf{f}_{c h}(\mathbf{x})\right)=$ $\left(a_{l u} f_{l u}(\mathbf{x}),\left|\mathbf{z}_{c h}\right| e^{i \varphi} \mathbf{f}_{c h}(\mathbf{x})\right)$ change luminance, hue and saturation of the initial image. The set of all such transformations forms the luminance-chromatic group $\mathbb{L} \mathbb{C} \mathbb{G}(2)=\left\{\left(a_{l u}, \mathbf{z}_{c h}\right) \mid\left(a_{l u} \in \mathbf{R}^{+}\right) \&\right.$ $\left.\left(\mathbf{z}_{c h} \in \mathbf{C}\right)\right\}$.

3.1.2 The Young-Helmholtz $k$-cycle model of multicolor images. We will interpret multicolor images (1) as multiplet-valued signals

$$
\mathbf{f}_{M c o l}(\mathbf{x})=f_{0}(\mathbf{x}) 1_{M c o l}+f_{1}(\mathbf{x}) \varepsilon_{M c o l}^{1}+\ldots+f_{k-1}(\mathbf{x}) \varepsilon_{M c o l}^{k-1}, \quad \mathbf{x} \in \mathbf{R}^{n},
$$

which take values in the multiplet $k$-cycle algebra

$$
\mathcal{A}_{k}^{M c o l}:=\mathbf{R} 1_{M c o l}+\mathbf{R} \varepsilon_{M c o l}^{1}+\ldots+\mathbf{R} \varepsilon_{M c o l}^{k-1},
$$


where $1, \varepsilon_{M c o l}^{1}, \ldots, \varepsilon_{M c o l}^{k-1}\left(\varepsilon_{M c o l}^{k}=1\right)$ are multicolor hyperimaginary units. We will denote them by $1, \varepsilon^{1}, \ldots, \varepsilon^{k-1}$. This algebra is called the multiplet (multicolor) algebra. One can show that this algebra is the direct sum of the real and complex fields:

$$
\mathcal{A}_{k}^{M c o l}=\sum_{i=1}^{k_{l u}}\left[\mathbf{R} \cdot \mathbf{e}_{l u}^{i}\right]+\sum_{j=1}^{k_{c h}}\left[\mathbf{C} \cdot \mathbf{E}_{c h}^{j}\right]=\mathbf{R}^{k_{l u}} \oplus \mathbf{C}^{k_{c h}}
$$

where $k_{l u}=1,2$ and $k_{c h}=\frac{k}{2}, \frac{k-1}{2}$ if $k$ is odd or even, respectively, and $\mathbf{e}_{l u}^{i}$ and $\mathbf{E}_{c h}^{j}$ are orthogonal idempotent units such that $\left(\mathbf{e}_{l u}^{i}\right)^{2}=\mathbf{e}_{l u}^{i}$, $\mathbf{e}_{i} \mathbf{e}_{j}=\mathbf{e}_{j} \mathbf{e}_{i},\left(\mathbf{E}_{c h}^{j}\right)^{2}=\mathbf{E}_{c h}^{j}, \mathbf{E}_{c h}^{i} \mathbf{E}_{c h}^{j}=\mathbf{E}_{c h}^{j} \mathbf{E}_{c h}^{i}$, and $\mathbf{e}_{l u}^{i} \mathbf{E}_{c h}^{j}=\mathbf{E}_{c h}^{j} \mathbf{e}_{l u}^{i}=0$, for all $i, j$. Every multiplet $\mathcal{C}$ can be represented as a linear combination of $k_{l u}$ "scalar" parts and $k_{c h}$ "complex" parts:

$$
\mathcal{C}=\sum_{i=1}^{k_{l u}}\left(a_{i} \cdot \mathbf{e}_{l u}^{i}\right)+\sum_{j=1}^{k_{c h}}\left(\mathbf{z}_{j} \cdot \mathbf{E}_{c h}^{j}\right) .
$$

The real numbers $a_{i} \in \mathbf{R}$ are called the multi-intensity numbers and complex numbers $\mathbf{z}_{j}=b+i c \in \mathbf{C}$ are called the multi-chromacity numbers. Now we will interpret the multicolor $n \mathrm{D}$ image appearing on the $n \mathrm{D}$ retina as a multiplet-valued $n \mathrm{D}$ signal of the form:

$$
\begin{gathered}
\mathbf{f}_{M c o l}^{R e t}(\mathbf{x})=\sum_{i=1}^{k_{l u}}\left[f_{l u}^{i}(\mathbf{x}) \cdot \mathbf{e}_{l u}^{i}\right]+\sum_{j=1}^{k_{c h}}\left[f_{c h}^{j}(\mathbf{x}) \cdot \mathbf{E}_{c h}^{j}\right]= \\
\left(f_{l u}^{1}(\mathbf{x}), \ldots, f_{l u}^{k_{l u}}(\mathbf{x}) ; f_{c h}^{1}(\mathbf{x}), \ldots, f_{c h}^{k_{c h}}(\mathbf{x})\right) .
\end{gathered}
$$

Here, the argument $\mathbf{x}$ belongs to the $n \mathrm{D}$ vector part $\mathcal{G} \mathcal{R}_{n}^{p, q, r}$ of the space algebra $\mathcal{A}_{2^{n}}^{S p}$. Changes in both the surrounding world and mental spaces of reality, such as multi-intensity and multi-color, can be treated in the language of multiplet algebra as the action of the following transformation group in the perceptual multicolor space $\mathcal{A}_{k}^{M c o l}$. Letting

$$
\begin{gathered}
\mathcal{A}=\left(a_{l u}^{1}, \ldots, a_{l u}^{k_{l u}}, \mathbf{z}_{c h}^{1}, \ldots, \mathbf{z}_{c h}^{k_{c h}}\right)= \\
\left(a^{1}, \ldots, a^{k_{l u}} ;\left|\mathbf{z}_{c h}^{1}\right| e^{i \varphi_{1}},\left|\mathbf{z}_{c h}^{2}\right| e^{i \varphi_{2}}, \ldots,\left|\mathbf{z}_{c h}^{k_{c h}}\right| e^{i \varphi_{k h}}\right),
\end{gathered}
$$

the following transformations of a multicolor image

$$
\begin{gathered}
\mathbf{f}_{M c o l}(\mathbf{x}) \longrightarrow \mathcal{A} \mathbf{f}_{M c o l}(\mathbf{x})= \\
\left(a^{1} f_{l u}^{1}(\mathbf{x}), \ldots, a^{k_{l u}} f_{l u}^{k_{l u}}(\mathbf{x}) ;\left|\mathbf{z}_{c h}^{1}\right| e^{i \varphi_{1}} f_{c h}^{1}(\mathbf{x}), \ldots,\left|\mathbf{z}_{c h}^{k_{c h}}\right| e^{i \varphi_{k h}} f_{c h}^{k_{c h}}(\mathbf{x})\right)
\end{gathered}
$$


change multi-luminancies, multi-hues and multi-saturations of the initial multicolor image. The set of all such transformations forms the multiluminance/ multi-chromatic group $\mathbb{M L} \mathbb{C} \mathbb{G}(k):=\left\{\left(a_{l u}^{1}, \ldots, a_{l u}^{k_{l u}} ; \mathbf{z}_{c h}^{1}, \ldots\right.\right.$, $\left.\left.\mathbf{z}_{c h}^{k_{c h}}\right) \mid a_{l u}^{i} \in \mathbf{R}^{+}, \mathbf{z}_{c h}^{j} \in \mathbf{C}\right\}$.

\subsubsection{Multiorthounitary transforms of multicolor images.}

A 2D discrete multicolor image can be defined as a $2 \mathrm{D}$ array $\mathbf{f}_{M c o l}^{R e t}=$ $\left[\mathbf{f}_{\text {Mcol }}^{\text {Ret }}(i, j)\right]_{i, j=1}^{N}$, i.e., as a $2 \mathrm{D}$ discrete $\mathcal{A}_{k}^{M c o l}$-valued function of one of the following forms:

$$
\mathbf{f}_{\text {col }}^{\text {Ret }}(i, j): \mathbf{Z}_{N}^{2} \longrightarrow \mathcal{A}_{k}^{M c o l}, \quad \mathbf{f}_{c o l}^{R e t}(i, j): \mathbf{Z}_{N}^{2} \longrightarrow \mathbf{R}^{k_{l u}} \oplus \mathbf{C}^{k_{c h}} .
$$

Here, every color pixel $\mathbf{f}_{M c o l}^{R e t}(i, j)$ at position $(i, j)$ is a multicolor number of the type $\mathbf{f}_{\text {Mcol }}^{\text {Ret }}(i, j)=f_{0}(i, j) 1_{M c o l}+f_{1}(i, j) \varepsilon_{M c o l}^{1}+\ldots+f_{k-1}(i, j) \varepsilon_{M c o l}^{k-1}$ or of the type $\left.\mathbf{f}_{M c o l}^{R e t}(i, j)\right)=\sum_{i=1}^{k_{l u}}\left[f_{l u}^{i}(i, j) \cdot \mathbf{e}_{l u}^{i}\right]+\sum_{j=1}^{k_{c h}}\left[f_{c h}^{j}(i, j) \cdot \mathbf{E}_{c h}^{j}\right]=$ $\left(f_{l u}^{1}(i, j), \ldots, f_{l u}^{k_{l u}}(i, j) ; f_{c h}^{1}(i, j), \ldots, f_{c h}^{k_{c h}}(i, j)\right)$. In particular, for color images, we have $\mathbf{f}_{c o l}^{\text {Ret }}(i, j)=f_{r}(i, j)+f_{g}(i, j) \varepsilon^{1}+f_{b}(i, j) \varepsilon^{2}$ and $\mathbf{f}_{\text {col }}^{\text {Ret }}(i, j)=$ $f_{l u}(i, j) \mathbf{e}_{l u}+\mathbf{f}_{c h}(i, j) \mathbf{E}_{c h}$. The set of all such images forms $N^{2} \mathrm{D}$ GreavesHilbert space $\left(\mathcal{A}_{k}^{M c o l}\right)^{N^{2}}$. The vector space structure of this space is defined with multiplication by triplet-valued scalars $(\mathcal{C} f)(i, j):=\bigodot e f(i, j)$. Our $N^{2} \mathrm{D}$ Greaves-Hilbert space $\left(\mathcal{A}_{k}^{M c o l}\right)^{N^{2}}$ over $\mathcal{A}_{k}^{M c o l}$ can be interpreted as $k N^{2} \mathrm{D}$ Hilbert space over $\mathbf{R}$, i.e., as $\left(\mathcal{A}_{k}^{M c o l}\right)^{N^{2}}=\mathbf{R}^{N^{2}} 1+$ $\mathbf{R}^{N^{2}} \varepsilon^{1}+\ldots+\mathbf{R}^{N^{2}} \varepsilon^{k-1}$, or as a direct sum of $N^{2} \mathrm{D}$ real and complex Hilbert spaces

$$
\left(\mathcal{A}_{k}^{M c o l}\right)^{N^{2}}=\left[\bigoplus_{i=1}^{k_{l u}} \mathbf{R}^{N^{2}} \mathbf{e}_{l u}^{i}\right]+\left[\bigoplus_{j=1}^{k_{c h}} \mathbf{C}^{N^{2}} \mathbf{E}_{c h}^{j}\right]=\left[\bigoplus_{i=1}^{k_{l u}} \mathbf{R}^{N^{2}}\right] \oplus\left[\bigoplus_{j=1}^{k_{c h}} \mathbf{C}^{N^{2}}\right] .
$$

In particular, for color images $\left(\mathcal{A}_{3}^{\text {col }}\right)^{N^{2}}=\mathbf{R}^{N^{2}}+\mathbf{R}^{N^{2}} \varepsilon^{1}+\mathbf{R}^{N^{2}} \varepsilon^{2}$, or $\left(\mathcal{A}_{3}^{c o l}\right)^{N^{2}}=\mathbf{R}^{N^{2}} \oplus \mathbf{C}^{N^{2}}$, where $\mathbf{R}^{N^{2}}, \mathbf{R}^{N^{2}} \varepsilon^{1}, \mathbb{R}^{N^{2}} \varepsilon^{2}$ are real $N^{2}$ Hilbert spaces of red, green, and blue images, respectively, $\mathbf{R}^{N^{2}}$ is $N^{2} \mathrm{D}$ real space of gray-level images, and $\mathbf{C}^{N^{2}}$ is $N^{2} \mathrm{D}$ complex space of chromatic images. Let $\left(\mathcal{A}_{k}^{M c o l}\right)^{N^{2}}$ be a $N^{2}$ Greaves-Hilbert space over $\mathcal{A}_{k}^{M c o l}$. We say that the operator $\mathfrak{L}_{2 D}:\left(\mathcal{A}_{k}^{M c o l}\right)^{N^{2}} \rightarrow\left(\mathcal{A}_{k}^{M c o l}\right)^{N^{2}}, \mathfrak{L}_{2 D}\left[\mathbf{f}_{M c o l}^{\text {Ret }}\right]=$ $\mathbf{F}_{\text {Mcol }}^{\text {Ret }}$ is multicolor linear if and only if for all $\mathbf{f}_{\text {Mcol }}^{\text {Ret }}, \mathbf{g}_{\text {Mcol }}^{\text {Ret }} \in\left(\mathcal{A}_{k}^{M c o l}\right)^{N^{2}}$ and for all $\mathcal{C} \in \mathcal{A}_{k}^{\text {Mcol }} \mathfrak{L}_{2 D}\left[\mathbf{f}_{\text {Mcol }}^{\text {Ret }}+\mathbf{g}_{\text {Mcol }}^{\text {Ret }}\right]=\mathfrak{L}_{2 D}\left[\mathbf{f}_{\text {Mcol }}^{\text {Ret }}\right]+\mathfrak{L}_{2 D}\left[\mathbf{g}_{\text {Mcol }}^{\text {Ret }}\right]$, $\mathfrak{L}_{2 D}\left[\mathcal{C} \mathbf{f}_{\text {Mcol }}^{\text {Ret }}\right]=\mathcal{C} \mathfrak{L}_{2 D}\left[\mathbf{f}_{\text {Mcol }}^{\text {Ret }}\right]$. Otherwise, we call $\mathfrak{L}_{2 D}$ multicolor nonlinear. If $\mathfrak{L}_{2 D}$ is a multicolor linear operator and $\left\|\operatorname{det}\left(\mathfrak{L}_{2 D}\right)\right\|_{2}^{2}$ does not 
vanish, then we call operator $\mathfrak{L}_{2 D}$ nonsingular. Otherwise, we call $\mathfrak{L}_{2 D}$ singular. The inverse $\mathfrak{L}_{2 D}^{-1}$ of an operator $\mathfrak{L}_{2 D}$ exists if and only if $\mathfrak{L}_{2 D}$ is nonsingular. $\mathfrak{L}_{2 D}^{-1}$ is calculated in the same way as an ordinary inverse matrix. The set of nonsingular multicolor linear operators form the general multicolor linear groups over multiplet algebra $\mathcal{A}_{k}^{\text {Mcol }}$ and is denoted by $\mathbf{G L}\left(N, \mathcal{A}_{k}^{M c o l}\right)$. It is then easy to define the adjoint operator $\mathfrak{L}_{2 D}^{*}$ for the color linear operator $\mathfrak{L}_{2 D}$ whose essential properties are $\left\langle\mathbf{f}_{\text {Mcol }}^{\text {Ret }} \mid \mathfrak{L}_{2 D} \mathbf{g}_{\text {Mcol }}^{\text {Ret }}\right\rangle=\left\langle\mathfrak{L}_{2 D}^{*} \mathbf{f}_{\text {Mcol }}^{\text {Ret }} \mid \mathbf{g}_{\text {Mcol }}^{\text {Ret }}\right\rangle$, and for ${ }_{1} \mathfrak{L}_{2 D}{ }_{2} \mathfrak{L}_{2 D}$ the property $\left({ }_{1} \mathfrak{L}_{2 D} \mathfrak{L}_{2 D}\right)^{*}={ }_{2} \mathfrak{L}_{2 D}^{*} \mathfrak{L}_{2 D}^{*}$ is true.

Definition 1 A multicolor linear operator $\mathfrak{L}_{2 D}$ on $\left(\mathcal{A}_{k}^{M c o l}\right)^{N^{2}}$ is said to be multi-orthounitary if $\mathfrak{L}_{2 D}^{-1}=\mathfrak{L}_{2 D}^{*}$.

Multi-orthounitary operators form the multi-orthounitary group $\mathbb{M O U}$ $=\operatorname{MOU}\left(\mathcal{A}_{k}^{M c o l}\right)$. This group is isomorphic to the direct sum of $k_{l u}$ orthogonal and $k_{c h}$ unitary groups, $\mathbb{M O U}\left(\mathcal{A}_{k}^{M c o l}\right)=$

$$
\left[\bigoplus_{i=1}^{k_{l u}} \mathbb{O}(\mathbf{R}) \mathbf{e}_{l u}^{i}\right]+\left[\bigoplus_{j=1}^{k_{c h}} \mathbb{U}(\mathbf{C}) \mathbf{E}_{c h}^{j}\right]=\left[\bigoplus_{i=1}^{k_{l u}} \mathbb{O}(\mathbf{R})\right] \oplus\left[\bigoplus_{j=1}^{k_{c h}} \mathbb{U}(\mathbf{C})\right] .
$$

In particular, the orthounitary group of transformations for color images has the decomposition: $\mathbb{O} \mathbb{U}\left(\mathcal{A}_{3}^{\text {col }}\right)=\mathbb{O}(\mathbf{R}) \mathbf{e}_{l u}+\mathbb{U}(\mathbf{C}) \mathbf{E}_{c h}$. Every element of $\mathbb{M O U}\left(\mathcal{A}_{k}^{M c o l}\right)$ and $\mathbb{O U}\left(\mathcal{A}_{3}^{\text {col }}\right)$ has the representation:

$$
\mathfrak{L}_{2 D}=\left[\bigoplus_{i=1}^{k_{l u}} \mathbf{O}_{2 D}^{i} \mathbf{e}_{l u}^{i}\right]+\left[\bigoplus_{j=1}^{k_{c h}} \mathcal{U}_{2 D}^{j} \mathbf{E}_{c h}^{j}\right], \quad \mathfrak{L}_{2 D}=\mathbf{O}_{2 D} \mathbf{e}_{l u}+\mathcal{U}_{2 D} \mathbf{E}_{c h}
$$

where $\mathbf{O}_{2 D}^{i} \in \mathbb{O}^{i}(\mathbf{R}), \mathbf{O}_{2 D} \in \mathbb{O}(\mathbf{R})$, and $\mathcal{U}_{2 D}^{j} \in \mathbb{U}^{j}(\mathbf{C}) \mathcal{U}_{2 D} \in \mathbb{U}(\mathbf{C})$ are orthogonal and unitary transforms, respectively. For multicolor image processing we shall use separable $2 D$ transforms.

Definition 2 We call the multi-orthounitary transform $\mathfrak{L}_{2 D}\left[\mathbf{f}_{\text {Mcol }}^{\text {Ret }}\right]$ separable if it can be represented by $\mathfrak{L}_{2 D}\left[\mathbf{f}_{\text {Mcol }}^{\text {Ret }}\right]=\mathfrak{L}_{1 D}\left[\mathbf{f}_{\text {Mcol }}^{\text {Ret }}\right] \mathfrak{M}_{1 D}$, i.e., $\mathfrak{L}_{2 D}=\mathfrak{L}_{1 D} \otimes \mathfrak{M}_{1 D}$ is the tensor product of two $1 D$ multi-orthounitary transforms of the form:

$$
\mathfrak{L}_{2 D}=\mathfrak{L}_{1 D} \otimes \mathfrak{L}_{1 D}=\left[\bigoplus_{i=1}^{k_{l u}}\left(\mathbf{O}_{1}^{i} \otimes \mathbf{O}_{2}^{i}\right) \mathbf{e}_{l u}^{i}\right]+\left[\bigoplus_{j=1}^{k_{c h}}\left(\mathcal{U}_{1}^{j} \otimes \mathfrak{U}_{2}^{j}\right) \mathbf{E}_{c h}^{j}\right]
$$

for multicolor images, and the tensor product of two $1 D$ orthounitary transforms $\mathfrak{L}_{2 D}=\mathfrak{L}_{1 D} \otimes \mathfrak{L}_{1 D}=\left(\mathbf{O}_{1} \otimes \mathbf{O}_{2}\right) \mathbf{e}_{l u}+\left(\mathcal{U}_{1} \otimes \mathfrak{U}_{2}\right) \mathbf{E}_{\text {ch }}$ for color images, where $\mathbf{O}_{1}^{i}, \mathbf{O}_{2}^{i}$ and $\mathcal{U}_{1}^{j}, \mathcal{U}_{2}^{j}$ are $1 D$ orthogonal and unitary transforms, respectively. 


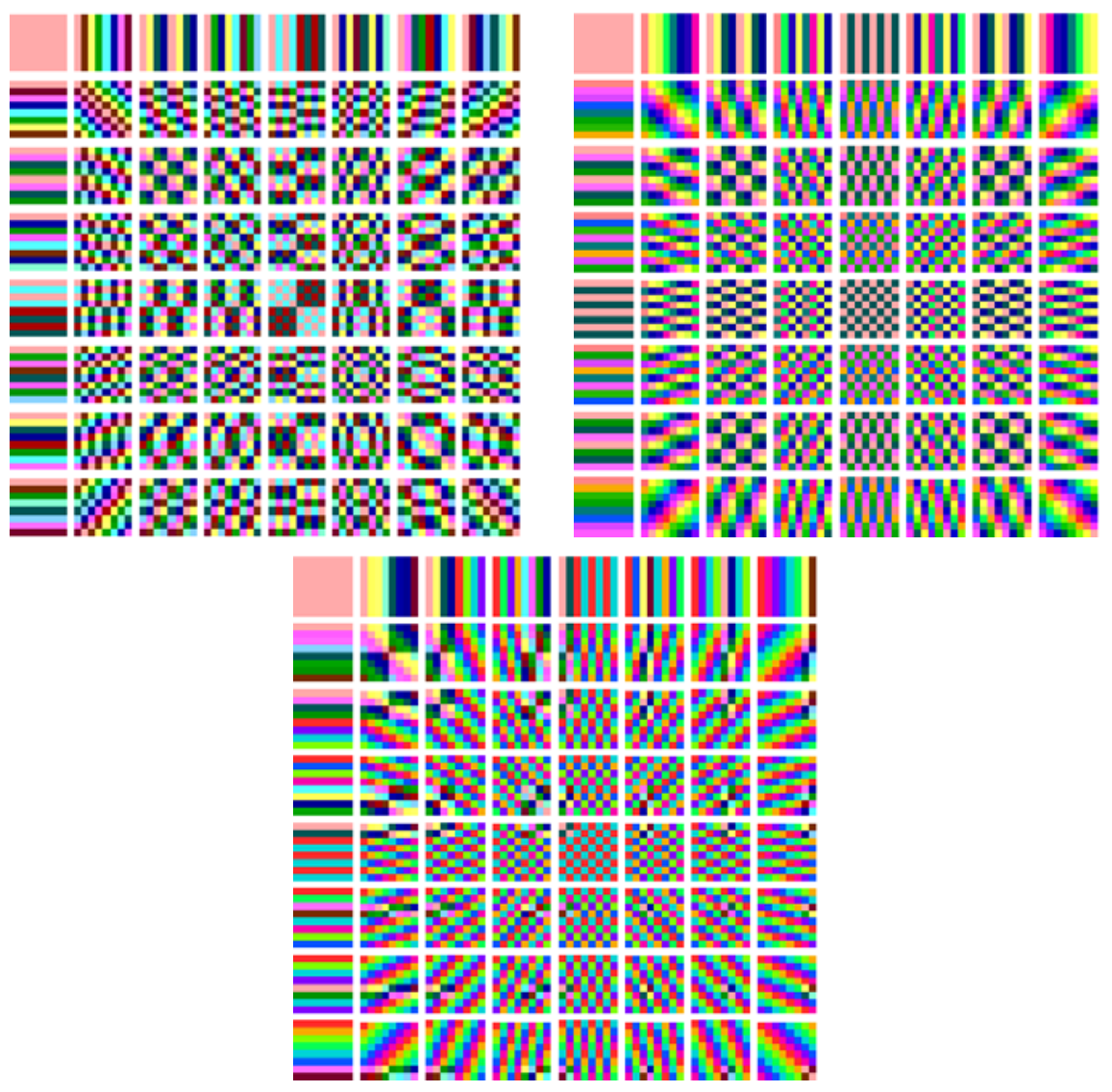

Figure 5. Color Walsh-Fourier $\mathfrak{W} \mathfrak{F}=\mathbf{W e}_{l u}+\mathcal{F} \mathbf{E}_{c h}$, Hartley-Fourier $\mathfrak{H} \mathfrak{T} \mathfrak{F}=$ $\mathbf{H t e}_{l u}+\mathcal{F} \mathbf{E}_{c h}$ and Haar-Fourier $\mathfrak{H} \mathfrak{R F}=\mathbf{H r e}_{l u}+\mathcal{F} \mathbf{E}_{c h}$ transforms, respectively

In particular, we can obtain any orthounitary transform, using any two pairs of orthogonal $\mathbf{O}_{1}, \mathbf{O}_{2}$ and unitary transforms $\mathcal{U}_{1}, \mathcal{U}_{2}$. It is possible to use one pair of orthogonal and unitary transforms, when $\mathbf{O}_{1}=\mathbf{O}_{2}=\mathbf{O}$ and $\mathcal{U}_{1}=\mathcal{U}_{2}=\mathcal{U}$. In this case we obtain a wide family of orthounitary transforms of the form: $\mathfrak{L}_{2 D}=(\mathbf{O} \otimes \mathbf{O}) \mathbf{e}_{l u}+(\mathcal{U} \otimes \mathcal{U}) \mathbf{E}_{c h}$, using different $1 \mathrm{D}$ orthogonal transforms. The following table shows 
some of the possibilities:

\begin{tabular}{|c|c|c|c|c|}
\hline & $\mathcal{F}$ & $\mathrm{eW}$ & eFP & $\mathcal{C H}$ \\
\hline $\mathbf{W}$ & $\overline{\mathbf{W} e_{l u}+\mathcal{F} \mathbf{E}_{c h}}$ & $\mathbf{W} e_{l u}+\mathcal{C W} \mathbf{E}_{c h}$ & $\mathbf{W} e_{l u}+\mathcal{C} \mathcal{F P} \mathbf{E}_{c h}$ & $\overline{\mathbf{W} e_{l u}+\mathcal{C} \mathcal{H} \mathbf{E}_{c h}}$ \\
\hline Hd & $\overline{\mathbf{H d} e_{l u}+\mathcal{F} \mathbf{E}_{c h}}$ & $\mathbf{H d} e_{l u}+\mathcal{C} \mathcal{W} \mathbf{E}_{c h}$ & $\mathbf{H d} e_{l u}+\mathcal{C F P} \mathbf{E}_{c h}$ & $\overline{\mathbf{H d}} e_{l u}+\mathcal{C} \mathcal{H} \mathbf{E}_{c h}$ \\
\hline $\mathbf{H t}$ & $\mathbf{H t} e_{l u}+\mathcal{F} \mathbf{E}_{c h}$ & $\mathbf{H t} e_{l u}+\mathcal{C W} \mathbf{E}_{c h}$ & $\mathbf{H t}_{l u}+\mathcal{C F P} \mathbf{E}_{c h}$ & $\mathbf{H t} e_{l u}+\mathcal{C H} \mathbf{E}_{c h}$ \\
\hline $\mathbf{H r}$ & $\mathbf{H r} e_{l u}+\mathcal{F} \mathbf{E}_{c h}$ & $\mathbf{H r} e_{l u}+\mathcal{C W} \mathbf{E}_{c h}$ & $\mathbf{H r} e_{l u}+\mathcal{C F P} \mathbf{E}_{c h}$ & $\overline{\mathbf{H r}} e_{l u}+\mathcal{C} \mathcal{H} \mathbf{E}_{c h}$ \\
\hline$\overline{\mathbf{W v}}$ & $\overline{\mathbf{W v} e_{l u}+\mathcal{F} \mathbf{E}_{c h}}$ & $\mathbf{W v} e_{l u}+\mathcal{C} \mathcal{W} \mathbf{E}_{c h}$ & $\mathbf{W v}_{l u}+\mathcal{C F} \mathcal{F} \mathbf{E}_{c h}$ & $\mathbf{W v} e_{l u}+\mathcal{C} \mathcal{F} \mathbf{E}_{c h}$ \\
\hline
\end{tabular}

where $\mathbf{W}, \mathbf{H d}, \mathbf{H t}, \mathbf{H r}, \mathbf{W v}$ are Walsh, Hadamard, Hartley, Haar, and Wavelet orthogonal transforms, and $\mathcal{F}, \mathcal{C W}$, $\mathcal{E F P}$, and $\mathcal{C H}$ are Fourier, complex Walsh, complex Fourier-Prometheus, complex Haar transforms. Every pair $(\mathbf{O}, \mathbf{U})$ of an orthogonal $\mathbf{O}$ and a unitary $\mathcal{U}$ transform generates an orthounitary (color) transform $\mathfrak{L}=\mathbf{O e}_{l u}+\mathcal{U} \mathbf{E}_{c h}$. Some examples of basis color functions of color transforms $\mathfrak{W F}=\mathbf{W e}_{l u}+\mathcal{F} \mathbf{E}_{c h}$, $\mathfrak{H T F}=\mathbf{H t e}_{l u}+\mathcal{F} \mathbf{E}_{c h}, \mathfrak{H} \mathfrak{R F}=\mathbf{H r e}_{l u}+\mathcal{F} \mathbf{E}_{c h}$ are shown in Fig. 5.

\subsection{Algebraization of the Hering model}

3.2.1 The Hering Z/2Z-graded model of color images. Let us consider $3 \mathrm{D}$ color space $\mathbf{R}_{\text {col }}^{3}=\mathbf{R} J_{R}+\mathbf{R} J_{G}+\mathbf{R} J_{B}$ spanned by three new hyperimaginary units $J_{i}(i=1,2,3$ or $i=R, G, B): \quad J_{1}=J_{R}$ (red unit), $J_{2}=J_{G}$ (green unit), $J_{3}=J_{B}$ (blue unit). We assume $J_{R}^{2}=a_{r}, J_{G}^{2}=a_{g}, J_{B}^{2}=a_{b}$, where $a_{r}, a_{g}, a_{b} \in\{+1,0,-1\}$. We assume $J_{i}^{2}=+1$ for $i=1, \ldots, \alpha, J_{i}^{2}=-1$ for $i=\alpha+1, \ldots, \alpha+\beta, J_{i}^{2}=0$ for $i=\alpha+\beta+1, \ldots, \alpha+\beta+\gamma$, where $\alpha+\beta+\gamma=3$. Further, we construct a new color Clifford algebra $\mathcal{A}_{2^{3}}^{\operatorname{col}(\alpha, \beta, \gamma)}\left(J_{R}, J_{G}, J_{B}\right)=\mathbf{R} J_{B l}+$ $\left(\mathbf{R} J_{R}+\mathbf{R} J_{G}+\mathbf{R} J_{B}\right)+\left(\mathbf{R} J_{R G}+\mathbf{R} J_{R B}+\mathbf{R} J_{G B}\right)+\mathbf{R} J_{W h}$, where $J_{B l}=1$, $J_{W h}=J_{R} J_{G} J_{B}$ are black and white units, $I_{R G}:=J_{R} J_{G}, J_{R B}:=J_{R} J_{B}$, $J_{G B}:=J_{G} J_{B}$. This is an algebra of generalized color octonions with signature $(\alpha, \beta, \gamma)$.

Definition 3 The functions $\mathbf{f}_{\text {col }}^{V C}: \mathcal{G R}_{n}^{p, q, r} \rightarrow \mathcal{A}_{8}^{\operatorname{col}(\alpha, \beta, \gamma)}\left(J_{R}, J_{G}, J_{B}\right)$ of the form:

$$
\begin{aligned}
& \mathbf{f}_{c o l}^{V C}(\mathbf{x})=f_{B l}(\mathbf{x}) J_{\emptyset}+\left(f_{R}(\mathbf{x}) J_{R}+f_{G}(\mathbf{x}) J_{G}+f_{B}(\mathbf{x}) J_{B}\right)+ \\
& +\left(f_{R G}(\mathbf{x}) J_{R G}+f_{R B}(\mathbf{x}) J_{R B}+f_{G B}(\mathbf{x}) J_{G B}\right)+f_{W h}(\mathbf{x}) J_{W h}
\end{aligned}
$$

are called the $\mathcal{A}_{2^{3}}^{\operatorname{col}(\alpha, \beta, \gamma)}$-valued color $n \mathrm{D}$ images appearing in the human Visual Cortex.

The second opponent cells map R,G,B components on the 4D unit sphere $f_{B l}^{2}+f_{R G}^{2}+f_{R B}^{2}+f_{G B}^{2}=1$, where $f_{R G}, f_{R B}, f_{G B}, f_{B l}$ are black, red-green, 
red-blue and green-blue components, respectively. Therefore, resulting from the capacity of this algebraic model of color image, we can formulate the spin-valued function

$$
\mathbf{f}_{c o l}^{V C}(\mathbf{x}):=f_{B l}(\mathbf{x}) J_{B l}+\left(f_{R G}(\mathbf{x}) J_{R G}+f_{R B}(\mathbf{x}) J_{R B}+f_{G B}(\mathbf{x}) J_{G B}\right)
$$

which has values in the spin part $\operatorname{Spin}\left(\mathcal{A}_{8}^{c o l)}\left(J_{R}, J_{G}, J_{B}\right)\right)$ of the color Clifford algebra.

DeFinition 4 The functions $\mathbf{f}_{\text {col }}^{V C}: \mathbf{R}^{2} \longrightarrow \operatorname{Spin}\left(\mathcal{A}_{8}^{c o l}\left(J_{R}, J_{G}, J_{B}\right)\right)$, and $\mathbf{f}_{\text {col }}^{V C}: \mathbf{R}^{n} \longrightarrow \operatorname{Spin}\left(\mathcal{A}_{8}^{\text {col }}\left(J_{R}, J_{G}, J_{B}\right)\right)$ are called the spin-valued color $2 \mathrm{D}$ and $n \mathrm{D}$ images, respectively.

For this model, color changes in both the surrounding world and mental spaces can be described as the action of the color spinor transformation group in the perceptual color spaces $\mathcal{A}_{8}^{\text {col }}$ and $\operatorname{Spin}\left(\mathcal{A}_{8}^{\text {col }}\right)$. If $\mathcal{C}_{0} \in \operatorname{Spin}\left(\mathcal{A}_{8}^{c o l}\right)$, then the transformations $\mathbf{f}_{c o l}^{V C}(\mathbf{x}) \longrightarrow \mathcal{C}_{0} \mathbf{f}_{c o l}^{V C}(\mathbf{x})$, $\mathbf{f}_{c o l}^{V C}(\mathbf{x}) \longrightarrow \mathbf{f}_{c o l}^{V C}(\mathbf{x}) \mathcal{C}_{0}^{-1}$ and $\mathbf{f}_{c o l}^{V C}(\mathbf{x}) \longrightarrow \mathcal{C}_{0} \mathbf{f}_{c o l}^{V C}(\mathbf{x}) \mathcal{C}_{0}^{-1}$ are called the left, right and two-sided spinor-color transformations of $\mathbf{f}_{c o l}^{V C}(\mathbf{x})$, respectively.

\subsubsection{The Hering $\mathrm{Z} / \mathrm{kZ}$-graded model of multicolor images.}

To form the algebraic model of multicolor images in the animals' VC, we consider a $k^{m} \mathrm{D}$ multicolor quantum Clifford algebra

$$
\mathcal{Q} \mathcal{A} \mathcal{A}_{k^{m}}^{M \operatorname{col}\left(\alpha_{0}, \alpha_{1}, \ldots, \alpha_{k-1}\right)}\left(J_{1}, \ldots, J_{m}\right)
$$

(i.e., Clifford algebra with signature $\left(\alpha_{0}, \alpha_{1}, \ldots, \alpha_{k-1}\right)$ and deformed by a $k$ primitive root of unity $\omega_{k}$ ) generated by multicolor hypercomplex units $J_{1}, \ldots, J_{m}$ with relations $J_{i}^{k}=\omega^{0}$ for $\alpha_{0}$ hypercomplex units, $J_{i}^{k}=\omega^{1}$ for $\alpha_{1}$ hypercomplex units, $\ldots, J_{i}^{k}=\omega^{k-1}$ for $\alpha_{k-1}$ hypercomplex units, and $J_{J} J_{i}=\omega_{k} J_{i} J_{j}$, if $i<j$, where $\alpha_{0}+\alpha_{1}+\ldots+$ $\alpha_{k-1}=m, \omega:=\sqrt[k]{1}[9]$. The elements $\mathbf{J}^{\mathbf{s}}=J_{1}^{s_{1}} \cdots J_{m}^{s_{m}}$ form a basis of $\mathcal{Q} \mathcal{C} \mathcal{A}_{k^{m}}^{M \operatorname{col}\left(\alpha_{0}, \alpha_{1}, \ldots, \alpha_{k-1}\right)}\left(J_{1}, \ldots, J_{m}\right)$, where $\mathbf{s}=\left(s_{1}, \ldots, s_{m}\right) \in \mathbf{B}_{k}^{m}$, $s_{i} \in \mathbf{B}_{k}=\{0, \ldots, k-1\}$. We shall denote this algebra by $\mathcal{Q} \mathcal{C} \mathcal{A}_{k^{m}}^{M c o l}$, if $J_{1}, \ldots, J_{m}$, and $\left(\alpha_{0}, \alpha_{1}, \ldots, \alpha_{k-1}\right)$ are fixed. In the particular case when $k=2\left(\omega_{k}=-1\right), m=3$ and $\alpha_{0}=\alpha, \alpha_{1}=\beta, \alpha_{2}=\gamma$, the $k^{m} \mathrm{D}$ quantum Clifford algebra $\mathcal{Q} \mathcal{C} \mathcal{A}_{k^{m}}^{M \operatorname{ccol}\left(\alpha_{0}, \alpha_{1}, \ldots, \alpha_{k-1}\right)}\left(J_{1}, \ldots, J_{m}\right)$ is the generalized color octonion algebra $\mathcal{A}_{8}^{\operatorname{col}(\alpha, \beta, \gamma)}$. We can make $\mathcal{Q} \mathcal{C} \mathcal{A}_{k^{m}}^{M c o l}$ be a ranked and $\mathbf{Z} / k \mathbf{Z}$-graded algebra. Let $r(\mathbf{s})$ be the Hamming weight of $\mathbf{s}$, i.e., 
a functional $r: \mathbf{B}_{k}^{m} \longrightarrow[0, m-1]$ defined by $r(\mathbf{b}):=\sum_{i=1}^{m}\left[1-\delta_{0, s_{i}}\right]$, and let $\partial(\mathbf{s})=\sum_{i=1}^{m} s_{i}(\bmod k)$ be the $\operatorname{grad}$ of $\mathbf{s}$. We set $\mathcal{Q} \mathcal{C} \mathcal{A}_{k^{m}}^{[r]}=$ $\operatorname{SPAN}\left\{\mathbf{J}^{\mathbf{s}} \mid r(\mathbf{s})=r\right\}$, obtaining the ranked sum

$$
\mathcal{Q} \mathcal{C} \mathcal{A}_{k^{m}}^{M c o l}=\bigoplus_{r=0}^{m} \mathcal{Q} \mathcal{C} \mathcal{A}_{k^{m}}^{[r]}
$$

By setting $\mathcal{Q} \mathcal{C} \mathcal{A}_{k^{m}}^{\{l\}}=\mathbf{S P A N}\left\{\mathbf{J}^{\mathbf{s}} \mid \partial(\mathbf{s}) \equiv l \bmod k\right\}$, we get the $\mathbf{Z} / k \mathbf{Z}$ graded R-algebra $\mathcal{Q} \mathcal{C} \mathcal{A}_{k^{m}}^{M c o l}=\bigoplus_{i=0}^{k-1} \mathcal{Q} \mathcal{C} \mathcal{A}_{k^{m}}^{\{l\}}$. We put $\partial(\mathcal{C})=l$ and say that $l$ is the degree of $\mathcal{C}$. . We say that $\Phi_{g}$ and $\Phi_{r}$ are graded and ranked automorphisms if $\Phi_{g}\left(\mathcal{Q} \mathcal{C} \mathcal{A}_{k^{m}}^{\{l\}}\right)=\mathcal{Q} \mathcal{C} \mathcal{A}_{k^{m}}^{\{l\}}$ and $\Phi_{r}\left(\mathcal{Q} \mathcal{C} \mathcal{A}_{k^{m}}^{[r]}\right)=$ Qe $\mathcal{A}_{k^{m}}^{[r]}$ for all $l=0,1, \ldots, k-1$, and $r=0,1, \ldots, m$, respectively. Let $\mathcal{Q C}_{\mathcal{A}}^{[1]} k^{m}=\operatorname{SPAN}\left\{\mathbf{J}^{\mathbf{s}} \mid r(\mathbf{s})=1\right\}$ be the vector part of the quantum Clifford algebra $Q \mathcal{C} \mathcal{A}_{k^{m}}^{M c o l}$. Then the set of automorphisms of unit modulus $\Phi_{r}\left(\mathcal{Q} \mathcal{C} \mathcal{A}_{k^{m}}^{[1]}\right)=\mathcal{Q} \mathcal{C} \mathcal{A}_{k^{m}}^{[1]}$ forms a transformation group of $\mathcal{L} \mathcal{C} \mathcal{A}_{k^{m}}^{[1]}$. We shall call it the quantum spinor group and denote it by

$$
\operatorname{QSpin}\left(\mathcal{Q} \mathcal{C} \mathcal{A}_{k^{m}}^{M c o l}\right)
$$

DEFINITION 5 The functions

$$
\begin{gathered}
\mathbf{f}_{M \operatorname{Vcl}}^{V C}(\mathbf{x}): \mathcal{G R}_{n}^{S p(p, q, r)} \longrightarrow \mathcal{Q} \mathcal{C} \mathcal{A}_{k^{m}}^{M \operatorname{col}\left(\alpha_{0}, \alpha_{1}, \ldots, \alpha_{k-1}\right)}\left(J_{1}, \ldots, J_{m}\right), \\
\mathbf{f}_{M \operatorname{Vcol}}^{V C}(\mathbf{x}): \mathcal{G} \mathcal{R}_{n}^{S p(p, q, r)} \longrightarrow \operatorname{QSpin}\left(\mathcal{Q} \mathcal{A}_{k^{m}}^{M \operatorname{col}\left(\alpha_{0}, \alpha_{1}, \ldots, \alpha_{k-1}\right)}\left(J_{1}, \ldots, J_{m}\right)\right)
\end{gathered}
$$

are called the $n D$ Cliffordean-valued and quantum-spin-valued images appearing in the animals' $V C$.

For this model, multicolor changes in both the surrounding world and mental spaces can be described as the action of the quantum spinor transformation group in the perceptual color spaces $\mathrm{QSpin}\left(\mathcal{Q} \mathcal{C} \mathcal{A}_{k^{m}}^{M c o l}\right)$ and $\mathcal{Q} \mathcal{C} \mathcal{A}_{k^{m}}^{M c o l}$. If $\mathcal{C}_{0} \in \mathrm{QSpin}\left(\mathcal{Q} \mathcal{C} \mathcal{A}_{k^{m}}^{M c o l}\right)$, then the transformations

$$
\begin{gathered}
\mathbf{f}_{M c o l}^{V C}(\mathbf{x}) \longrightarrow \mathcal{C}_{0} \mathbf{f}_{M c o l}^{V C}(\mathbf{x}) \\
\mathbf{f}_{M c o l}^{V C}(\mathbf{x}) \longrightarrow \mathbf{f}_{M c o l}^{V C}(\mathbf{x}) \mathcal{C}_{0}^{-1} \quad \text { and } \quad \mathbf{f}_{M c o l}^{V C}(\mathbf{x}) \longrightarrow \mathcal{C}_{0} \mathbf{f}_{M c o l}^{V C}(\mathbf{x}) \mathcal{C}_{0}^{-1}
\end{gathered}
$$


are called the left, right and two-sided spinor-color transformations of $\mathbf{f}_{M c o l}^{V C}(\mathbf{x})$, respectively. Further, we interpret an image as an embedding of a manifold in a spatial-multicolor Clifford algebra of higher dimension. The embedding manifold is a "hybrid" (spatial-multicolor) space that includes spatial coordinates as well as color coordinates. For example, a $2 \mathrm{D}$ color image is considered as a $3 \mathrm{D}$ manifold in the $5 \mathrm{D}$ spatial-color space $\mathbf{R}_{S p C o l}^{5}\left(I_{1}, I_{2} ; J_{R}, J_{G}, J_{B}\right)=\left(\mathbf{R} I_{1}+\mathbf{R} I_{2}\right) \oplus\left(\mathbf{R} J_{R}+\right.$ $\left.\mathbf{R} J_{G}+\mathbf{R} J_{B}\right)=\mathbf{R}_{S p}^{2} \oplus \mathbf{R}_{c o l}^{3}$, whose coordinates are $\left(x, y, f_{R}, f_{G}, f_{B}\right)$, where $x \in \mathbf{R} I_{1}, y \in \mathbf{R} I_{2}$ are spatial coordinates and $f_{R} \in \mathbf{R} J_{R}$, $f_{G} \in \mathbf{R} J_{G}, f_{B} \in \mathbf{R} J_{B}$, are color coordinates. It is clear that the geometrical, color and spatial-multicolor spaces $\mathbf{R}_{S p}^{n}, \mathbf{R}_{M c o l}^{k}, \mathbf{R}_{S p M c o l}^{n+m(k-1)}$ generate spatial, color and quantum spatial-multicolor Clifford algebras $\mathcal{A}_{2^{n}}^{S p(p, q, r)}, \quad \mathcal{Q} \mathcal{A}_{k^{m}}^{M \operatorname{col}\left(\alpha_{0}, \alpha_{1}, \ldots, \alpha_{k-1}\right)}, \quad \mathcal{Q} \mathcal{A}_{2^{n} k^{m}}^{S p M \operatorname{col}\left(p, q, r ; \alpha_{0}, \alpha_{1}, \ldots, \alpha_{k-1}\right)}$, respectively. Here, all spatial hyperimaginary units commute with all multicolor units.

\subsubsection{Clifford-unitary transforms of multicolor images.}

2D discrete Cliffordean-valued images appearing in the animals' VC can be defined as a $2 \mathrm{D}$ array $\mathbf{f}_{M c o l}^{V C}:=\left[\mathbf{f}_{M c o l}^{V C}(i, j)\right]_{i, j=1}^{N}$. Here, every pixel $\mathbf{f}_{M c \text { col }}^{V C}(i, j)$ at position $(i, j)$ is a quantum Clifford number of the type $\mathbf{f}_{M c o l}^{V C}(i, j)=\sum_{\mathbf{s} \in \mathbf{B}_{k}^{m}} f_{\mathbf{s}}(i, j) \mathbf{J}^{\mathbf{s}}$. The set of all such images forms the $N^{2} \mathrm{D}$ Clifford-Hilbert space $\mathbb{L}\left(\mathbf{Z}_{N}^{2}, \mathcal{Q} \mathcal{C} \mathcal{A}_{k^{m}}^{M c o l}\right)=\left(\mathcal{Q} \mathcal{C} \mathcal{A}_{k^{m}}^{M c o l}\right)^{N^{2}}$. The vector structure of this space is defined with multiplication by Clifford-valued scalars $(\mathcal{C} f)(i, j):=\mathcal{e} f(i, j)$. We say that the operator

$$
\mathfrak{L}_{2 D}:\left(\mathcal{Q} \mathcal{\mathcal { A } _ { k ^ { m } } ^ { M c o l }}\right)^{N^{2}} \longrightarrow\left(\mathcal{Q} \mathcal{C} \mathcal{A}_{k^{m}}^{M c o l}\right)^{N^{2}}, \quad \text { i.e., } \quad \mathfrak{L}_{2 D}\left[\mathbf{f}_{M c o l}^{V C}\right]=\mathbf{F}_{M c o l}^{V C}
$$

is Clifford linear if and only if for all $\mathbf{f}_{\text {Mcol }}^{V C}, \mathbf{g}_{\text {Mcol }}^{V C} \in\left(2 \mathcal{C} \mathcal{A}_{k^{m}}^{M c o l}\right)^{N^{2}}$ and for all $\mathcal{C}_{1}, \mathcal{C}_{2}, \mathcal{B}_{1}, \mathcal{B}_{2} \in \mathcal{Q} \mathcal{C} \mathcal{A}_{k^{m}}^{M c o l}$

$$
\mathfrak{L}_{2 D}\left[\mathcal{C}_{1} \mathbf{f}_{M c o l}^{V C} \mathcal{B}_{1}+\mathcal{C}_{2} \mathbf{g}_{M c o l}^{V C} \mathcal{B}_{2}\right]=\mathcal{C}_{1} \mathfrak{L}_{2 D}\left[\mathbf{f}_{\text {Mcol }}^{V C}\right] \mathcal{B}_{1}+\mathfrak{C}_{2} \mathfrak{L}_{2 D}\left[\mathbf{g}_{M c o l}^{V C}\right] \mathcal{B}_{2} .
$$

Otherwise we call $\mathfrak{L}_{2 D}$ Clifford nonlinear. If $\mathfrak{L}_{2 D}$ is a Clifford linear operator and $\left\|\operatorname{det}\left(\mathfrak{L}_{2 D}\right)\right\|_{2}^{2}$ does not vanish, then we call the operator $\mathfrak{L}_{2 D}$ nonsingular. Otherwise $\mathfrak{L}_{2 D}$ is singular. The inverse $\mathfrak{L}_{2 D}^{-1}$ of the operator $\mathfrak{L}_{2 D}$ exists if and only if $\mathfrak{L}_{2 D}$ is nonsingular. The set of nonsingular Clifford linear operators forms the general Clifford linear groups over the quantum Clifford algebra $Q \mathcal{C} \mathcal{A}_{k^{m}}^{M c o l}$ and is denoted by $\mathbb{G} \mathbb{C L}\left(N, \mathcal{Q} \mathcal{A}_{k^{m}}^{M c o l}\right)$. It is then easy to define the adjoint operator $\mathfrak{L}_{2 D}^{*}$ 
for the Clifford linear operator $\mathfrak{L}_{2 D}$, whose essential properties are

$$
\left\langle\mathbf{f}_{M c o l}^{V C} \mid \mathfrak{L}_{2 D} \mathbf{g}_{M c o l}^{V C}\right\rangle=\left\langle\mathfrak{L}_{2 D}^{*} \mathbf{f}_{M c o l}^{V C} \mid \mathbf{g}_{M c o l}^{V C}\right\rangle,
$$

and for the product ${ }_{1} \mathfrak{L}_{2 D} \mathfrak{L}_{2 D}$ we have

$$
\left({ }_{1} \mathfrak{L}_{2 D} \mathfrak{L}_{2 D}\right)^{*}={ }_{2} \mathfrak{L}_{2 D}^{*} \mathfrak{L}_{2 D}^{*} .
$$

The Clifford linear operator $\mathfrak{L}_{2 D}$ is said to be Clifford-unitary if

$$
\mathfrak{L}_{2 D}^{-1}=\mathfrak{L}_{2 D}^{*}
$$

Clifford-unitary operators form the Clifford-unitary multicolor group $\mathbb{C U}\left(N, \mathcal{Q} \mathcal{C} \mathcal{A}_{k^{m}}^{M c o l}\right)$. For multicolor image processing we shall use separable $2 D$ transforms. The Clifford-unitary transform $\mathfrak{L}_{2 D}\left[\mathbf{f}_{M c o l}^{V C}\right]=\mathbf{F}_{M c o l}^{V C}$ is called separable if it can be represented by $\mathbf{F}_{\text {Mcol }}^{V C}=\mathfrak{L}_{2 D}\left[\mathbf{f}_{M c o l}^{V C}\right]=$ $\mathfrak{L}_{1 D}\left[\mathbf{f}_{M c o l}^{V C}\right] \mathfrak{M}_{1 D}$, i.e., $\mathfrak{L}_{2 D}=\mathfrak{L}_{1 D} \otimes \mathfrak{M}_{1 D}$ is the tensor product of two $1 D$ Clifford-unitary transforms.

\section{Hypercomplex-valued invariants of $n \mathrm{D}$ multicolor images}

\subsection{Clifford-valued invariants}

Let us assume that $\mathbf{f}_{M c o l}(\mathbf{x}): \mathbf{R}_{S p}^{n} \longrightarrow \mathcal{A}_{k}^{M c o l}$ is an image of a multicolor $n \mathrm{D}$ object. It appears on the $n \mathrm{D}$ eye retina as a function $\mathbf{f}_{M c o l}^{\text {Ret }}(\mathbf{x})$ of space variables $\mathbf{x} \in \operatorname{Vec}^{1}\left(\mathcal{A}_{2^{n}}^{S p}\right)=\mathcal{G} \mathcal{R}_{n}^{S p(p, q, r)}$ with values in the multicolor algebra $\mathcal{A}_{k}^{M c o l}: \mathbf{f}_{M c o l}^{R e t}(\mathbf{x}): \mathcal{G R}_{n}^{S p(p, q, r)} \longrightarrow \mathcal{A}_{k}^{M c o l}$. This image can be considered in the $\mathrm{VC}$ as a function $\mathbf{f}_{M c o l}^{V C}(\mathbf{x})$ of the same space variables $\mathbf{x}$, but with values in the multicolor quantum Clifford algebra $\mathcal{Q} \mathcal{C} \mathcal{A}_{k^{n}}^{M c o l}$, i.e., as $\mathbf{f}_{M c o l}^{V C}(\mathbf{x}): \mathcal{G R}_{n}^{S p(p, q, r)} \longrightarrow \mathcal{Q} \mathcal{C} \mathcal{A}_{k^{n}}^{M c o l}$. We shall denote both algebras $\mathcal{A}_{k}^{M c o l}$ and $\mathcal{Q} \mathcal{C} \mathcal{A}_{k^{n}}^{M c o l}$ by $\mathcal{A}^{M c o l}$. Changes in the surrounding world can be treated in the language of the spatial-multicolor algebra as an action of two groups: the space affine group $\operatorname{Aff}\left(\mathcal{G R}_{n}^{p, q, r}\right)$ acting on the physical space $\operatorname{Vec}^{1}\left(\mathcal{A}_{2^{n}}^{S p}\right)=\mathcal{G R}_{n}^{p, q, r}$ and the multi-color group $\mathbb{M L} \mathbb{C} G(k)$ acting on $\mathcal{A}_{k}^{M c o l}$ (if $\mathcal{A}^{\text {Mcol }}=\mathcal{A}_{k}^{M c o l}$ ) or the quantum spin group $\operatorname{Spin}\left(\mathcal{Q} \mathcal{C} \mathcal{A}_{k^{m}}^{M c o l}\right)$ on $\mathscr{Q} \mathcal{C} \mathcal{A}_{k^{n}}^{M c o l}$ (if $\mathcal{A}^{M c o l}=\mathcal{Q} \mathcal{C} \mathcal{A}_{k^{n}}^{M c o l}$ ). We shall denote both groups $\operatorname{Spin}\left(\mathcal{Q} \mathcal{C} \mathcal{A}_{k^{m}}^{M c o l}\right)$ and $\mathbb{M L} \mathbb{C} G(k)$ by $\mathcal{M} \mathcal{C} \mathcal{G} \mathcal{R}^{M c o l}$. Let $\mathbf{G}^{S p M c o l}=\operatorname{Aff}\left(\mathcal{G R}_{n}^{S p(p, q, r)}\right) \times \mathcal{M} \mathcal{M} \mathcal{G} \mathcal{R}^{M c o l}$ be a spatial-multicolor group, and $\left(\mathbf{g}^{S p}, \mathbf{g}^{M c o l}\right) \in \mathbf{G}^{S p M c o l}$, where $\mathbf{g}^{S p} \in \mathbf{A f f}\left(\mathcal{G R}_{n}^{p, q, r}\right), \mathbf{g}^{M c o l} \in$ $\mathcal{M C G R}^{M c o l}$. If $\mathrm{x} \in \mathcal{G R}_{n}^{S p(p, q, r)}$ is a generalized space Clifford number and $\mathcal{C} \in \mathcal{A}^{M c o l}$ is a multicolor quantum Clifford number, then all products of the form $\mathrm{x} \mathcal{C}$ are called spatial-color numbers. They form a spacecolor algebra $\mathcal{A}^{S p M c o l}:=\mathcal{A}_{2^{n}}^{S p} \otimes \mathcal{A}^{M c o l}$. Here, we assume that all spatial hyperimaginary units commute with all color units. 
Definition 6 The $\mathcal{A}^{\text {SpMcol }}$-valued functional $\mathcal{J}=\mathcal{M}\left[\mathbf{f}_{M c o l}(\mathbf{x})\right]$ of the image $\mathbf{f}_{M c o l}(\mathbf{x})$ is called the relative $\mathbf{G}^{\text {SpMcol-invariant if }}$

$$
\mathcal{J}=\mathcal{M}\left\{\mathbf{g}^{M c o l} \circ \mathbf{f}_{M c o l}\left(\mathbf{g}^{S p} \circ \mathbf{x}\right)\right\}=\mathcal{C} \cdot \mathcal{M}\left\{\mathbf{f}_{M c o l}(\mathbf{x})\right\} \cdot \mathcal{C}^{-1},
$$

$\forall \mathbf{g} \in \mathbf{G}^{\text {SpMcol }}$, where $\mathcal{C}, \mathrm{C}^{-1}$ are left and right $\mathcal{A}^{\text {SpMcol_valued multipliers. }}$ If $\mathrm{C}=1$ then $\mathrm{J}$ is called the absolute invariant and denoted by $\mathrm{J}$.

Let $\mathbf{c}$ be the centroid of the image $\mathbf{f}_{M c o l}(\mathbf{x})$.

DEFINITION 7 The functionals

$$
\mathcal{M}_{p}:=\mathcal{M}\left\{\mathbf{f}_{M c o l}\right\}=\int_{\mathbf{x} \in \mathcal{G} \mathcal{R}_{n}^{S p}}(\mathbf{x}-\mathbf{c})^{p} \mathbf{f}_{M c o l}(\mathbf{x}) \mathbf{d} \mathbf{x}
$$

are called the central $\mathcal{A}^{S p M c o l}$-valued moments of the $n D$ image $\mathbf{f}_{M c o l}(\mathbf{x})$, where $p \in \mathbf{Q}$ are rational numbers.

Let us clarify the rules of moment transformations with respect to distortions of color and geometry of the initial images. If $\mathbf{f}_{M c o l}(\mathbf{x})$ is the initial image, then $\mathbf{f}_{\lambda, \mathcal{E}_{0}, \mathbf{w}}^{\mathcal{C}_{0}}\left(\mathbf{x}^{*}\right)=\mathcal{C}_{0}\left\{\mathbf{f}_{M c o l}\left(\lambda \mathcal{E}_{0}(\mathbf{x}+\mathbf{w}) \mathcal{E}_{0}^{-1}\right)\right\} \mathcal{C}_{0}^{-1}$ denotes its $\mathcal{A}^{M c o l}$-multicolor and $\mathcal{G R}_{n}^{p, q, r}$-geometrical distorted copy. Here $\mathbf{v}, \mathbf{w}$ are $n \mathrm{D}$ vectors. Summing $\mathbf{v}$ with $\mathbf{w}$ brings us to image translation by the vector $\mathbf{w}$, two-sided multiplications $\lambda \varepsilon_{0}(\mathbf{x}+\mathbf{w}) \mathcal{E}_{0}^{-1}$ by $\lambda \varepsilon_{0}$ and $\mathcal{E}_{0}^{-1}$ equivalent to both an $n \mathrm{D}$ rotation of the vector $\mathbf{z}+\mathbf{w}$ and a dilatation given by the factor $\lambda$. Here, $\mathbf{f}_{M c o l} \longrightarrow \mathcal{C}_{0} \mathbf{f}_{M c o l} \mathcal{C}_{0}^{-1}$ is a multicolor transformation of the initial image.

THEOREM 2 The central moments $\mathcal{M}_{p}$ of the multicolor images $\mathbf{f}_{M c o l}(\mathbf{x})$ are relative $\mathcal{A}^{\text {SpMcol}}$-valued invariants

$$
\mathcal{J}_{p}\left\{\lambda \varepsilon_{0} \mathbf{w} \mathbf{f}_{M c o l}^{\mathcal{C}_{0}}\right\}=\mathcal{M}_{p}\left\{\lambda \varepsilon_{0} \mathbf{W} \mathbf{f}_{M c o l}^{\mathfrak{C}_{0}}\right\}=\left(\lambda^{p+n} \mathcal{C}_{0} \mathcal{E}_{0}^{p}\right) \mathcal{M}_{p}\left\{\mathbf{f}_{M c o l}\right\}\left(\mathcal{E}_{0}^{-p} \mathcal{C}_{0}^{-1}\right)
$$

with respect to the spatial-multicolor group $\mathbf{G}^{\text {SpMcol }}$ with both $\mathcal{A}^{\text {SpMcol }}$ valued left multipliers $\mathcal{C}_{0} \lambda^{p+3} \mathcal{E}_{0}^{p}$ and $\mathcal{A}_{2^{n}}^{S p}$-valued right multipliers $\mathcal{E}_{0}^{-p}$, respectively (see Fig. 6), and the normalized central moments

$$
\left|\mathcal{N}_{p}\left\{{ }_{\lambda \varepsilon_{0} \mathbf{w}} \mathbf{f}_{M c o l}^{\mathcal{C}_{0}}\right\}\right|=\left|\mathcal{M}_{p}\left\{\lambda \varepsilon_{0} \mathbf{w} \mathbf{f}_{M c o l}^{\mathfrak{C}_{0}}\right\} \mathcal{M}_{0}^{p-1}\left\{{ }_{\lambda \varepsilon_{0} \mathbf{w}} \mathbf{f}_{M c o l}^{\mathcal{C}_{0}}\right\}\right| /\left|\mathcal{M}_{1}^{p}\left\{{ }_{\lambda \varepsilon_{0} \mathbf{w}} \mathbf{f}_{M c o l}^{\mathfrak{C}_{0}}\right\}\right|
$$

are absolute scalar-valued invariants, with respect to the same group, i.e., $\mathcal{J}_{p}=\left|\mathcal{N}_{p}\left\{\lambda \varepsilon_{0} \mathbf{w} \mathbf{f}_{\text {Mcol }}^{\mathcal{C}_{0}}\right\}\right|=\left|\mathcal{N}_{p}\left\{\mathbf{f}_{\text {Mcol }}\right\}\right|$.

Now we consider the most important cases for invariants of grey-level, color and multicolor 2D and 3D images. 


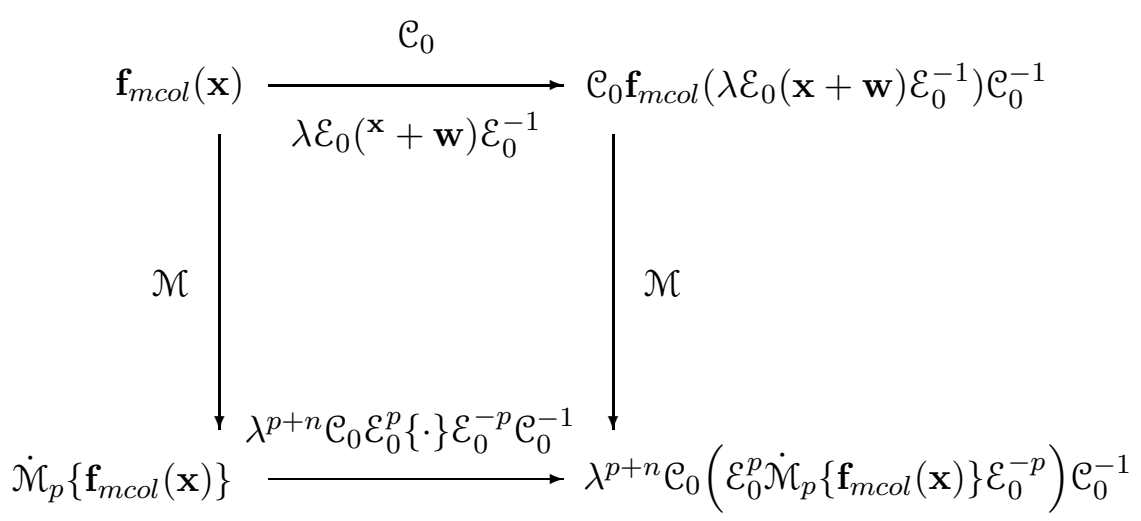

Figure 6. Transformations of $\mathcal{A}^{S p M c o l}$-valued moments with respect to the spatialmulticolor group $\mathbf{G}_{n, k}^{S p M c o l}$

\subsection{Complex and quaternion invariants of 2D and 3D grey-level images}

Let $\mathcal{G C}_{2}^{S p}=\left\{\mathbf{z}=x+I y \mid x, y \in \mathbf{R} ; I^{2}=-1,0,+1\right\}$ be a generalized spatial complex plane. Then a grey-level 2D image $f(x, y)$ can be considered as a function of a generalized complex variable, i.e., $f(x, y)=f(\mathbf{z})$, where $\mathbf{z}=x+I y \in \mathcal{G C}_{2}^{S p}$. Let $\mathbf{c}$ be the centroid of the image $f(\mathbf{z})$.

Definition 8 Functionals of the form

$$
\dot{\mathbf{m}}_{p}\{f\}=\int_{\mathbf{z} \in \mathcal{G C}_{2}^{S p}}(\mathbf{z}-\mathbf{c})^{p} f(\mathbf{z}) d \mathbf{z}
$$

are called the one-index central $\mathcal{A}_{2}^{S p}$-valued moments of the image $f(\mathbf{z})$, where $\mathbf{d} \mathbf{z}:=d x d y$, and $p \in \mathbf{Q}$ are rational numbers.

Let us clarify the rules of $\mathcal{A}_{2}^{S p}$-valued moment transformations under geometrical distortions of the initial $2 \mathrm{D}$ images. We will consider translation, rotation and scaling transformations. If $f(\mathbf{z})$ is the initial image, then $f_{\mathbf{v}, \mathbf{w}}(\mathbf{z})=f(\mathbf{v}(\mathbf{z}+\mathbf{w}))$ denotes its geometrical distorted copy.

THEOREM 3 The central moments $\dot{\mathbf{m}}_{p}\{f\}$ of the image $f(\mathbf{z})$ are relative $\mathcal{A}_{2}^{S p}$-valued invariants

$$
\partial_{p}\left\{f_{\mathbf{v}, \mathbf{w}}\right\}:=\dot{\mathbf{m}}_{p}\left\{f_{\mathbf{v}, \mathbf{w}}\right\}=\mathbf{v}^{p}|\mathbf{v}|^{2} \dot{\mathbf{m}}_{p}\{f\}
$$

with respect to the affine group $\mathbf{A f f}\left(\mathcal{G C}_{2}^{S p}\right)$ with $\mathcal{A}_{2}^{S p}$-valued multipliers $\mathbf{v}^{p}|\mathbf{v}|^{2}=e^{I p \varphi}|\mathbf{v}|^{p+2}$ (see Fig. 7), and the normalized central $\mathcal{A}_{2}^{S p}$ - 
valued moments $\mathcal{N}_{p}\left\{f_{\mathbf{v}, \mathbf{w}}\right\}:=\dot{\mathbf{m}}_{p}\left\{f_{\mathbf{v}, \mathbf{w}}\right\} \dot{\mathbf{m}}_{0}^{p-1}\left\{f_{\mathbf{v}, \mathbf{w}}\right\} / \dot{\mathbf{m}}_{1}^{p}\left\{f_{\mathbf{v}, \mathbf{w}}\right\}$ are absolute $\mathcal{A}_{2}^{S p}$-valued invariants with respect to the same group, i.e., $\mathcal{J}_{p}=$ $\mathcal{N}_{p}\left\{f_{\mathbf{v}, \mathbf{w}}\right\}=\mathcal{N}_{p}\{f\}$.

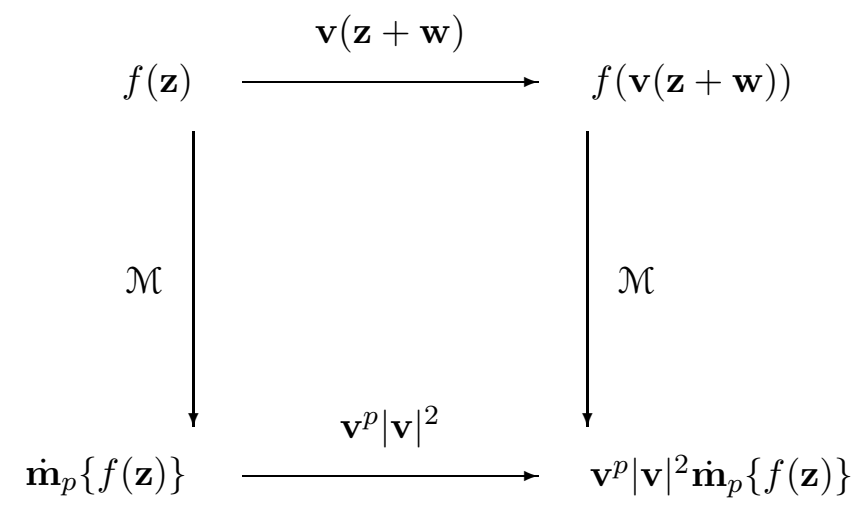

Figure \%. Transformations of $\mathcal{A}_{2}^{S p}$-valued moments with respect to the affine group $\operatorname{Aff}\left(\mathcal{G e}_{2}^{S p}\right)$

Let us consider a 3D grey-level image $f(x, y, z)$. This image can be considered as a function of a generalized quaternion variable $\mathbf{q}=(x I+y J+$ $z K)$, i.e., $f(x, y, z)=f(\mathbf{q})$, where $\mathbf{q} \in \operatorname{Vec}\left\{\mathcal{A}_{4}^{S p}\right\}=\mathcal{G} \mathcal{R}^{3}$. Let $\mathbf{c}$ be the centroid of the image $f(\mathbf{q})$.

Definition 9 Functionals of the form

$$
\mathcal{M}_{p}\{f\}:=\int_{\mathbf{q} \in \mathcal{G R} \mathcal{R}^{3}}(\mathbf{q}-\mathbf{c}) f(\mathbf{q}) \mathbf{d} \mathbf{q}
$$

are called the one-index central $\mathcal{A}_{4}^{S p}$-valued (quaternion-valued) moments of the grey-level $3 D$ image $f(\mathbf{q})$, where $p \in \mathbf{Q}$, and $\mathbf{d q}:=d x d y d z$.

Let us clarify the rules of moment transformations with respect to geometrical distortions of 3D images. These distortions will be caused by 1) $3 \mathrm{D}$ translations $\mathbf{q} \longrightarrow \mathbf{q}+\mathbf{w}, 2) 3 \mathrm{D}$ rotations $\mathbf{q} \longrightarrow Q_{0}(\mathbf{q}+\mathbf{w}) Q^{-1}$, where $\left.Q_{0}=e^{\mathbf{u}_{0} \phi / 2}, 3\right)$ dilatation: $\mathbf{q} \longrightarrow \lambda \mathbf{q}$, where $\lambda \in \mathbf{R}^{+}$. If $f(\mathbf{q})$ is an initial image and $f_{\lambda Q_{0} \mathbf{w}}(\mathbf{q})$ its distorted version, then $f_{\lambda Q_{0} \mathbf{w}}(\mathbf{q}):=$ $f\left(\lambda Q_{0}(\mathbf{q}+\mathbf{w}) Q_{0}^{-1}\right)$.

THEOREM 4 The central moments $\mathcal{M}_{p}\{f\}$ of the $3 D$ grey-level image $f(\mathbf{q})$ are relative $\mathcal{A}_{4}^{S p}$-valued invariants

$$
\mathcal{J}_{p}\left\{f_{\lambda Q_{0} \mathbf{w}}\right\}:=\mathcal{M}_{p}\left\{f_{\lambda Q_{0} \mathbf{w}}\right\}=\left(\lambda^{p+3} Q_{0}^{p}\right) \mathcal{M}_{p}\{f\}\left(\mathcal{Q}_{0}^{-p}\right)
$$




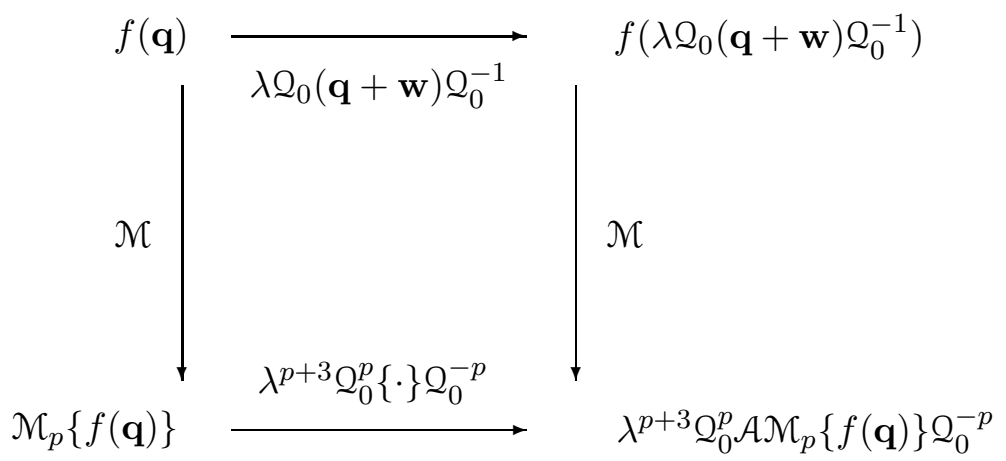

Figure 8. Transformations of $\mathcal{A}_{4}^{S p}$-valued moments with respect to the group $\operatorname{Aff}\left(\mathcal{G C}_{3}^{S p}\right)$

with respect to the group $\mathbf{A f f}\left(\mathcal{G e}_{3}^{S p}\right)$ with left $\mathcal{A}_{4}^{S p}$-valued multipliers $\lambda^{p+3} \mathrm{Q}_{0}^{p}$ and with right $\mathcal{A}_{4}^{S p}$-valued multipliers $Q_{0}^{-p}$, respectively (see Fig. 8), and the absolute values of normalized central moments

$$
\left|\mathcal{N}_{p}\right|=\left|\mathcal{M}_{p}\left\{f_{\lambda Q_{0} \mathbf{w}}(\mathbf{q})\right\} \mathcal{M}_{0}^{p-1}\left\{f_{\lambda Q_{0} \mathbf{w}}(\mathbf{q})\right\}\right| /\left|\mathcal{M}_{1}^{p-1}\left\{f_{\lambda Q_{0} \mathbf{w}}(\mathbf{q})\right\}\right|
$$

are absolute scalar-valued invariants with respect to the same group, i.e., $\mathcal{J}_{p}=\left|\mathcal{N}_{p}\left\{f_{\lambda Q_{0} \mathbf{w}}\right\}\right|=\left|\mathcal{N}_{p}\{f\}\right|$.

\subsection{Moments and invariants of color 2D and 3D images}

Let $\mathcal{G C}_{2}^{S p}:=\left\{\mathbf{z}=x_{1}+I x_{2} \mid x_{1}, x_{2} \in \mathbf{R} ; I^{2}=-1,0,+1\right\}$ be a generalized spatial complex plane. Then the color image $\mathbf{f}_{\text {col }}(x, y)$ can be considered as a triplet-valued function of the generalized complex variable $\mathbf{z}=x_{1}+I x_{2}$, i.e., as $\mathbf{f}_{c o l}(x, y)=\mathbf{f}_{\text {col }}(\mathbf{z})$, where $\mathbf{z} \in \mathcal{G C}_{2}^{S p}$. Let $\mathbf{z} \in \mathcal{G C}_{2}^{S p}$ be spatial and $\mathcal{A} \in \mathcal{A}_{3}^{C o l}$ be color triplet numbers. Then all products $\mathbf{z} \mathcal{A}$ will be called spatial-color numbers (or Hurwitz numbers). They form the 6D space-color Hurwitz algebra $\mathcal{A}_{6}^{S p C o l}:=\mathcal{A}_{2}^{S p} \otimes \mathcal{A}_{3}^{C o l}$. We assume that all spatial hyperimaginary units commute with all color units. Therefore, $\mathcal{A}_{6}^{S p C o l}=\mathcal{A}_{2}^{S p} \otimes \mathcal{A}_{3}^{C o l}=\mathcal{A}_{3}^{C o l} \otimes \mathcal{A}_{2}^{S p}$. Let $\mathbf{c}$ be the centroid of the image $\mathbf{f}_{\text {col }}(\mathbf{z})$.

Definition 10 Functionals of the form

$$
\dot{\mathcal{M}}_{p}=\int_{\mathbf{z} \in \mathcal{A}_{2}^{S p}}(\mathbf{z}-\mathbf{c})^{p} \mathbf{f}_{c o l}(\mathbf{z}) \mathbf{d} \mathbf{z}
$$


are called the central $\mathcal{A}_{6}^{\text {SpCol }}$-valued moments of the color image $\mathbf{f}_{\text {col }}(\mathbf{z})$.

Let $\mathcal{A}=\left(a_{l u}, \mathbf{z}_{c h}\right) \in \mathcal{A}_{3}^{C o l}$. Let us clarify the rules of moment transformations with respect to distortions of color and geometry of the initial images. If $\mathbf{f}_{\text {col }}(\mathbf{z})$ is the initial image, then $\mathbf{v}, \mathbf{w}_{\text {col }} \mathbf{f}^{\mathcal{A}}(z)=\mathcal{A} \mathbf{f}_{\text {col }}(\mathbf{v}(\mathbf{z}+\mathbf{w}))=$ $a_{l u} \mathbf{f}_{l u}(\mathbf{v}(\mathbf{z}+\mathbf{w})) \cdot \mathbf{e}_{l u}+\mathbf{z}_{c h} \mathbf{f}_{c h}(\mathbf{v}(\mathbf{z}+\mathbf{w})) \cdot \mathbf{E}_{c h}$ denotes its $\mathbb{L} \mathbb{C} \mathbb{G}(3)$-color and $\operatorname{Aff}\left(\mathcal{G C}_{2}^{S p}\right)$-geometrical distorted copy.

THEOREM 5 The central moments $\dot{\mathcal{M}}_{p}$ of the color image $\mathbf{f}_{\text {col }}(\mathbf{z})$ are relative $\mathcal{A}_{6}^{\text {SpCol }}$-valued invariants

$$
\mathcal{J}_{p}\left\{\mathbf{v}, \mathbf{w} \mathbf{f}_{c o l}^{\mathcal{A}}\right\}:=\dot{\mathcal{M}}_{p}\left\{\mathbf{v}, \mathbf{w} \mathbf{f}_{c o l}^{\mathcal{A}}\right\}=\mathcal{A} \mathbf{v}^{p}|\mathbf{v}|^{2} \dot{\mathcal{M}}_{p}\left\{\mathbf{f}_{c o l}\right\}
$$

with respect to the spatial-color group $\operatorname{Aff}\left(\mathcal{G C}_{2}^{S p}\right) \times \mathbb{L} \mathbb{C} G(3)$ with $\mathcal{A}_{6}^{S p C o l}$ valued multipliers $\mathcal{A} \mathbf{v}^{p}|\mathbf{v}|^{2}$ (see Fig. 9), and the absolute values of the normalized central moments

$$
\left|\dot{\mathcal{N}}_{p}\right|=\left|\dot{\mathcal{M}}_{p}\left\{\mathbf{v}, \mathbf{w} \mathbf{f}_{c o l}^{\mathcal{A}}\right\} \dot{\mathcal{N}}_{0}^{p-1}\left\{\mathbf{v}, \mathbf{w} \mathbf{f}_{c o l}^{\mathcal{A}}\right\}\right| /\left|\dot{\mathcal{M}}_{1}^{p}\left\{\mathbf{v}, \mathbf{w} \mathbf{f}_{c o l}^{\mathcal{A}}\right\}\right|
$$

are absolute scalar-valued invariants, with respect to the same group, i.e., $\mathcal{J}_{p}=\left|\dot{\mathcal{N}}_{p}\left\{\mathbf{v}, \mathbf{f}_{c o l}^{\mathcal{A}}\right\}\right|=\left|\dot{\mathcal{M}}_{p}\left\{\mathbf{f}_{c o l}\right\}\right|$.

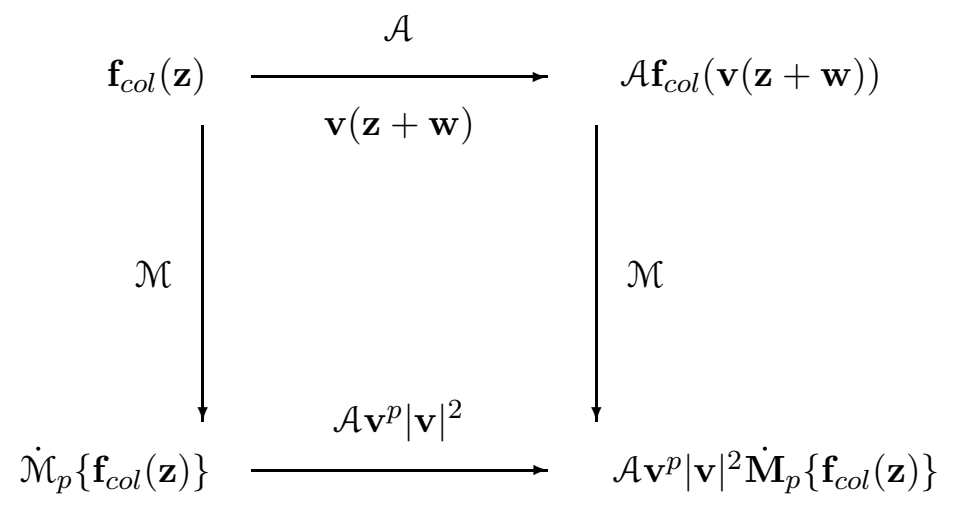

Figure 9. Transformations of relative $\mathcal{A}_{6}^{S p C o l}$-valued moments with respect to the spatial-color group $\mathbf{A f f}\left(\mathcal{G C}_{2}^{S p}\right) \times \mathbb{L C C G}(3)$

Let $\mathbf{f}_{c o l}(\mathbf{q})$ be a color 3D image depending on the pure vector generalized quaternion variable $\mathbf{q} \in \operatorname{Vec}\left\{\mathcal{A}_{4}^{S p}\right\}=\mathcal{G} \mathcal{R}^{3}$. If $\mathbf{q} \in \mathcal{C} \mathcal{R}_{3} \subset \mathcal{A}_{4}^{S p}$ is a generalized quaternion and $\mathcal{A} \in \mathcal{A}^{C o l}$ is a color number, then all products 
of the form $\mathbf{q} \mathcal{A}$ are called spatial-color quaternions. They form a spacecolor algebra $\mathcal{A}^{S p C o l}:=\mathcal{A}_{4}^{S p} \otimes \mathcal{A}^{C o l}=\mathcal{A}^{C o l} \otimes \mathcal{A}_{4}^{S p}$.

DEFinition 11 Functionals of the form

$$
\dot{\mathcal{M}}_{p}\left\{\mathbf{f}_{c o l}\right\}:=\int_{\mathbf{q} \in \mathcal{G} \mathcal{R}^{3}}(\mathbf{q}-\mathbf{c}) \mathbf{f}_{c o l}(\mathbf{q}) \mathbf{d} \mathbf{q}
$$

are called the one-index central $\mathcal{A}^{S p C o l}$-valued moments of the color $3 D$ image $\mathbf{f}_{\text {col }}(\mathbf{q})$, where $p \in \mathbf{Q}$, and $\mathbf{d q}:=d x d y d z$.

Let us clarify the rules of moment transformations with respect to geometrical and color distortions of $3 \mathrm{D}$ color images. If $f_{c o l}(\mathbf{q})$ is an initial image and ${ }_{\lambda \mathrm{Q}} \mathbf{w} \mathbf{f}_{\text {col }}^{\mathcal{A}}(\mathbf{q})$ its distorted version, then $\lambda_{\lambda \mathrm{Q}} \mathbf{f}_{c o l}^{\mathcal{A}}(\mathbf{q}):=$ $\mathcal{A} \mathbf{f}_{\text {col }}\left(\lambda Q(\mathbf{q}+\mathbf{w}) Q^{-1}\right)$.

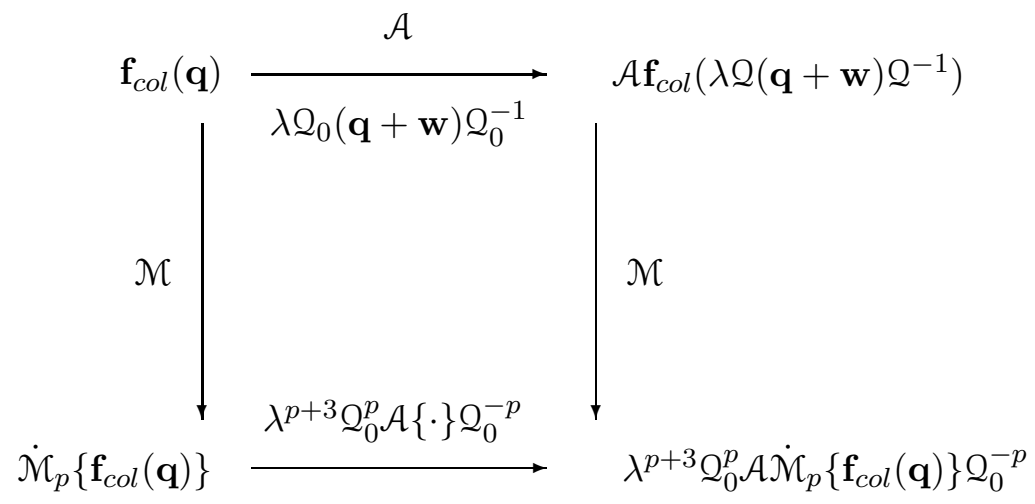

Figure 10. Transformations of $\mathcal{A}^{S p C o l}$-valued moments with respect to the spatialcolor group $\operatorname{Aff}\left(\mathcal{G e}_{3}^{S p}\right) \times \mathbb{L} \mathbb{C} G(3)$

THEOREM 6 The central moments $\dot{\mathcal{M}}_{p}\left\{\mathbf{f}_{\text {col }}\right\}$ of the 3-dimensional color image $f_{\text {col }}(\mathbf{q})$ are relative $\mathcal{A}^{S p C o l}$-valued invariants

$$
\partial_{p}\left\{\mathbf{f}_{\lambda Q_{0} \mathbf{w}}^{\mathcal{A}}\right\}:=\dot{\mathcal{M}}_{p}\left\{\mathbf{f}_{\lambda Q_{0} \mathbf{w}}^{\mathcal{A}}\right\}=\left(\mathcal{A} \lambda^{p+3} Q_{0}^{p}\right) \mathcal{M}_{p}\left\{\mathbf{f}_{c o l}\right\}\left(\mathcal{Q}_{0}^{-p}\right)
$$

with respect to the group $\mathbf{A f f}\left(\mathcal{G C}_{3}^{S p}\right) \times \mathbb{L} \mathbb{C} G(3)$ with left $\mathcal{A}^{S p C o l}$-valued multipliers $\mathcal{A} \lambda^{p+3} Q_{0}^{p}$ and with right $\operatorname{Spin}\left(\mathcal{A}_{4}^{S p}\right)$-valued multipliers $\mathcal{Q}_{0}^{-p}$, respectively (see Fig. 10), and the absolute values of the normalized central moments

$$
\left|\mathcal{N}_{p}\left\{\lambda \varepsilon_{0} \mathbf{w} \mathbf{f}_{c o l}^{\mathfrak{C}_{0}}\right\}\right|=\left|\mathcal{M}_{p}\left\{\lambda \varepsilon_{0} \mathbf{w} \mathbf{f}_{c o l}^{\mathfrak{C}_{0}}\right\} \mathcal{M}_{0}^{p-1}\left\{{ }_{\lambda \varepsilon_{0} \mathbf{w}} \mathbf{f}_{c o l}^{\mathfrak{C}_{0}}\right\}\right| /\left|\mathcal{M}_{1}^{p}\left\{\lambda \varepsilon_{0} \mathbf{w} \mathbf{f}_{c o l}^{\mathfrak{C}_{0}}\right\}\right|
$$


are relative scalar-valued invariants, with respect to the same group, i.e., $\mathcal{J}_{p}=\left|\mathcal{N}_{p}\left\{\lambda \varepsilon_{0} \mathbf{w} \mathbf{f}_{c o l}^{\mathfrak{C}_{0}}\right\}\right|=\left|\mathcal{N}_{p}\left\{\mathbf{f}_{c o l}\right\}\right|$.

\section{Conclusions}

We have shown how Clifford algebras can be used in the formation and computation of invariants of $2 \mathrm{D}, 3 \mathrm{D}$ and $n \mathrm{D}$ color and multicolor objects of different Euclidean and non-Euclidean geometries. The theorems stated show how simple and efficient the methods of calculation of invariants are that use spatial and color Clifford algebras. But how fast can the invariants be calculated? The answer to this question the interested reader can find in [2], where Fourier-Clifford and Fourier-Hamilton number theoretic transforms are used for this purpose.

\section{Acknowledgments}

The work was supported by the Russian Foundation for Basic Research, project no. 03-01-00735. The paper contains some results obtained in the project no.3258 of the Ural State Technical University. The author would like to thank Jim Byrnes, Director of the NATO Advanced Study Institute "Computational Noncommutative Algebra and Applications," and is grateful for his NATO support.

\section{References}

[1] Labunets-Rundblad, E.V., Labunets, V.G. and Astola, J. (1999). Is the Visual Cortex a Fast Clifford Algebra Quantum Compiler? A NATO Advanced Research Workshop Clifford Analysis and Its Applications, pp. 173-183.

[2] Labunets-Rundblad, E.V. (2000). Fast Fourier-Clifford Transforms Design and Application in Invariant Recognition. PhD thesis, Tampere University Technology, Tampere, Finland, 262 p.

[3] Labunets, V.G., Rundblad, E.V. and Astola, J., (2001). Is the brain "Clifford algebra quantum computer? Proc. of SPIE "Materials and Devices for Photonic Circuits", 2001 Vol. 4453, pp. 134-145

[4] Labunets, V.G., Rundblad, E.V. and Astola, J.,(2001). Fast invariant recognition of color 3D images based on spinor-valued moments and invariants. Proc. SPIE "Vision Geometry X", 2001, Vol. 4476, pp. 22-33

[5] Labunets, V.G., Maidan, A., Rundblad-Labunets, E.V. and Astola J., (2001). Colour triplet-valued wavelets and splines, Image and Signal Processing and Analysis ISPA'01, June 19-21, Pula, Croatia, 2001, pp. 535-541

[6] Labunets, V.G., Maidan, A., Rundblad-Labunets, E.V. and Astola J., (2001) Colour triplet-valued wavelets, splines and median filters, Spectral Methods and Multirate Signal Processing, SMMSP'2001, June 16-18, Pula, Croatia, 2001, pp. $61-70$

[7] Labunets-Rundblad, E.V. and Labunets, V.G. (2001). Chapter 7. Spatial-Colour Clifford Algebra for Invariant Image Recognition, pp. 155-185. (In: Geometric 
Computing with Clifford Algebra), Ed. G. Sommer. Springer, Berlin Heideberg, 2001, 452 p.

[8] Lasenby, A.N., Doran, C.J.L. and Gull, S.F. (1996). Lectures in Geometric Algebra, In: W. E. Baylis, Ed., Clifford (Geometric) Algebras with Applications to Physics, Mathematics and Engineering, Birkhouser, Boston, 256 p.

[9] Vela, M. (2000). Explicit solutions of Galois Embedding problems by means of generalized Clifford algebras, J. Symbolic Computation, 30, pp. 811-842

[10] Ch. Greaves, (1847). On algebraic triplets, Proc. Irish Acad., 3, pp. 51-54, 5764, 80-84, 105-108 